\title{
Spectroscopic Confirmation of Redshifts Predicted by Gravitational Lensing *
}

\author{
Tim Ebbels ${ }^{1}$, Richard Ellis ${ }^{1}$, Jean-Paul Kneib ${ }^{1}$, \\ Jean-François Le Borgne ${ }^{2}$, Roser Pelló ${ }^{2}$, Ian Smail ${ }^{3}$ \& Blai Sanahuja ${ }^{4}$ \\ ${ }^{1}$ Institute of Astronomy, Madingley Road, Cambridge CB3 OHA, U.K. \\ ${ }^{2}$ Observatoire Midi-Pyrénées, 14 Av. E.Belin, 31400 Toulouse, France. \\ ${ }^{3}$ Department of Physics, University of Durham, South Road, Durham. DH1 3LE \\ ${ }^{4}$ Departament d'Astronomia i Meteorologia, Universitat de Barcelona, Diagonal 648, 08028 Barcelona, Spain.
}

Accepted 1997 Received 1997 ; in original form

\begin{abstract}
We present deep spectroscopic measurements of 18 distant field galaxies identified as gravitationally-lensed arcs in a Hubble Space Telescope image of the cluster Abell 2218. Redshifts of these objects were predicted by Kneib et al. (1996) using a lensing analysis constrained by the properties of two bright arcs of known redshift and other multiply-imaged sources. The new spectroscopic identifications were obtained using long exposures with the LDSS-2 spectrograph on the William Herschel Telescope and demonstrate the capability of that instrument to new limits, $R \simeq 24$; the lensing magnification implies true source magnitudes as faint as $R \simeq 25$. Statistically, our measured redshifts are in excellent agreement with those predicted from Kneib et al.'s lensing analysis which gives considerable support to the redshift distribution derived by the lensing inversion method for the more numerous and fainter arclets extending to $R \simeq 25.5$. We explore the remaining uncertainties arising from both the mass distribution in the central regions of Abell 2218 and the inversion method itself, and conclude that the mean redshift of the faint field population at $R \simeq 25.5$ $(B \sim 26-27)$ is low, $\langle z\rangle=0.8-1$. We discuss this result in the context of redshift distributions estimated from multi-colour photometry. Although such comparisons are not straightforward, we suggest that photometric techniques may achieve a reasonable level of agreement particularly when they include near-infrared photometry with discriminatory capabilities in the $1<z<2$ range.
\end{abstract}

Key words: cosmology: observations - galaxies: evolution - gravitational lensing

\section{INTRODUCTION}

Gravitational lensing is now established as a highlysuccessful technique for studying both the mass distribution on various scales in rich clusters at intermediate depth, and for constraining the properties and distances of very faint sources magnified serendipitously via the lensing cluster. Smail et al. $(1994,1995 a)$ attempted to break the degeneracy between these two important applications by studying the lensing signal in a number of rich clusters chosen to lie at different redshifts. Although they concluded the bulk of a faint galaxy sample limited at $I \simeq 25$ has a redshift distribu-

\footnotetext{
* Based on observations obtained on the William Herschel Telescope at the Observatorio del Roque de los Muchachos, La Palma.
}

tion with a median close to one, their conclusion depended somewhat on the mass distribution in the more distant and hence less well-understood clusters.

The discovery of multiply-imaged systems in the cores of rich clusters (Mellier, Fort \& Kneib 1993, Kneib et al. 1993, Smail et al. 1995b) offers an alternative and more promising route. The mass models for clusters containing such multiple images are sufficiently constrained by the location and orientation of the images that it is possible to use these models to match the predicted shear of the fainter images with their observed shapes directly. In the case of multiple images, the requirement that each image must come from the same object in the source plane provides many constraints leading to a rather precise redshift prediction. Singly imaged arclets generate fewer constraints but nonetheless, 
a most likely redshift may be determined for each arclet, and useful statistical results can be obtained for the background redshift distribution, provided the sample size is large enough. A first application of this so-called 'lens inversion' technique was discussed by Kneib et al. (1994) for the cluster Abell 370. Individual redshifts were obtained for 30 candidate arclets limited at $B=27$ with axial ratios $a / b>1.4$ measured on ground-based images.

The inversion technique was considered in more detail by Kneib et al (1996, hereafter KESCS) on the basis of an impressive image of Abell $2218(z=0.175)$ obtained with the refurbished Hubble Space Telescope (HST), Figure 1. The important role of HST imaging in lensing studies was demonstrated in two respects. Firstly, via the superlative resolution of HST, the multiply imaged nature of several of the most strongly lensed arcs was revealed in spectacular detail. Secondly, in the absence of ground-based seeing, the fainter sheared images were individually identified with much greater confidence than was possible using groundbased data. This, in turn, enabled accurate inversion for a greater number of faint sources. Predicted redshifts were determined by KESCS for $\simeq 80$ arclets in Abell 2218 to $R=25$ and a formalism was developed to predict the uncertainties arising in the inversion from a number of sources. The limitations of lensing inversion as a general surveying technique were also discussed.

Although impressive progress has been made in locating high redshift galaxies via deep $(K<20)$ magnitude-limited redshift surveys (Cowie et al. 1995, 1996) and via various colour selection criteria such as those sensitive to the presence of a Lyman limit in the near ultraviolet (Steidel et al. 1996), an important advantage of lensing inversion as a purely geometric technique over other methods is that it does not require the presence of a particular spectral feature within some restricted observational passband. This suggests it should complement the other techniques by providing an accurate mean redshift distribution at various limits even if, ultimately, inversion through several clusters at various redshifts may be required to secure robust results.

Given the promise of the method it is clearly important to investigate the uncertainties of lensing inversion by direct spectroscopic measurement of the brighter arclets. This paper is concerned with executing such a test for Abell 2218 whose mass model is highly constrained from 7 multiple image sets, two of which already have spectroscopic redshifts (Pelló et al. 1992). Whereas only a few of the brighter arclets are amenable to direct spectroscopy with current facilities, the test should still be valuable since inversion is primarily a geometric technique. The estimated redshift for a particular arclet relies on the cluster mass distribution, the relative distances of the source and lens, and uncertainties in the source and image shape (whose contribution can be readily accounted for - see KESCS). Thus a comparison of the spectroscopic and predicted redshifts for the brighter arclets can be made as valid a test of the method as that, more difficult, test applied to the entire sample of arclets.

We have begun a survey of those arclets amenable for spectroscopic study with the $4.2 \mathrm{~m}$ William Herschel Telescope (WHT). Our aim is to verify the inversion technique and, if necessary, correct the residuals and produce an improved mass model. This in turn will lead to better inversion for the more numerous and fainter arclets. As part of this programme, one arclet in the sample (\#384 in the numbering scheme of Le Borgne et al. 1992) has already been discussed by Ebbels et al. (1996) owing to its high redshift $(z=2.515)$ and the spectacular agreement with the lensing prediction $\left(z=2.8_{-0.3}^{+0.5}\right)$, making it an object of interest in its own right. A plan of the paper follows. In $\S 2$ we discuss the selection criteria for our sample of arclets in Abell 2218 and present the journal of observations and spectroscopic results. In $\S 3$ we compare the observed redshifts with those predicted and consider the uncertainties. In $\S 4$ we review the mass model and illustrate those changes which could still be permitted whilst being consistent with the new sample of arclet redshifts. Finally, in $\S 5$ we return to the question of the mean redshift of the faint population following the discussion of KESCS. $\S 6$ summarizes our conclusions.

\section{OBSERVATIONS AND SPECTROSCOPIC REDSHIFTS}

The primary source material for our arclet sample is the HST WFPC2 image of A2218 as published by KESCS and shown in Figure 1. The three orbit exposure totaling 6500s was taken in the F702W passband and the magnitudes converted to standard $\mathrm{R}$ using corrections from Holtzman et al. (1995). The resulting object catalogue reaches $R=26$ with a completeness of $55 \%$ at $R \simeq 25$. Ground based $(B-I)$ colours for our targets were measured from images obtained with the COSMIC imaging spectrograph on the 5-m Hale telescope at Palomar during June 1994 and June 1995. A thick $2048^{2}$ TEK CCD (0.284 arcsec pix $\left.{ }^{-1}\right)$ was used and individual exposures of $\sim 500-1000$ s were taken using infield dithering (by $\sim 60$ arcsec). The images were reduced in a standard manner with IRAF using both twilight and onsource flat fields and including cosmic ray rejection. Stacked exposure times totaled $16.5 \mathrm{ks}$ in $B$ and $21.7 \mathrm{ks}$ in $I$, reaching $1 \sigma$ limiting surface brightnesses of $\mu_{B}=28.2$ and $\mu_{I}=26.8$ mag $\operatorname{arcsec}^{-2}$, while the final seeing on the frames measured $1.20 \operatorname{arcsec}$ in $B$ and $0.95 \operatorname{arcsec}$ in $I . B \& I$ magnitudes were measured from these deep exposures. Due to the faintness of these objects and the likelihood of contamination by nearby bright cluster galaxies, we measured these colours by hand. First, using IRAF's IMREPLACE task, a rectangular region enclosing the target object was replaced by fitting a surface to the perimeter pixels and then using the distribution of the perimeter pixel values to add sky noise to this surface. Then a difference image was formed between the original and object-replaced images such that only the flux from each object remained. Magnitudes were measured within circular apertures of radius 1 or 2 arcseconds and colours obtained from measurements within the same aperture in each band.

In selecting our arclet sample from the HST image, we employed three criteria. Firstly, we selected objects which looked distorted, indicating that they might be gravitational arclets. As in KESCS, we maximized the probability of selecting lensed images by restricting the sample to those images whose orientation lies within $45 \mathrm{deg}$ of the local shear direction as predicted by the mass model. Secondly, to maximize the probability of securing a reliable spectrum with measurable features, we applied an integrated magnitude limit of $B \simeq 25$ (corresponding to $R \simeq 24$ ) and gave highest 
priority to objects bluer than $(B-I) \simeq 2.2$. The former constraint corresponds to the faintest objects within reach of the LDSS-2 spectrograph on the $4.2 \mathrm{~m}$ WHT and the colour criterion is about half a magnitude bluer than the cluster E/S0 sequence and increases the probability of finding emission lines in the spectrum. Finally, we were mindful that spectroscopic redshifts for some arclets would be more important in the verification of the inversion method. The uncertainty for each arclet is a function of location, shape and redshift and thus by selecting those arclets for which the expected error is small, we can provide a more stringent test of inversion. These criteria were applied to 572 objects detected at 2 sigma above the sky in the WFPC2 image and produced a working catalogue of some 65 candidate arclets.

All observations were conducted with the LDSS-2 multiobject spectrograph (Allington-Smith et al. 1994) on the 4.2 metre William Herschel Telescope and are summarized in Table 11. In designing multi-slit masks, we were constrained by the fact that most of the highly-magnified arcs lie close to the cluster core. This caused problems with crowding since we insisted on a minimum slit length of 10 arcseconds to enable good sky subtraction. A second obvious limitation is the orientation and curvature of the arclets. Although with ground-based seeing only the giant arcs are noticeably nonlinear in form, the orientations of the larger arclets must be taken into account in order to maximize the amount of light captured by each slit. Each mask had one primary target of this type and thus we were able to base the mask orientation on the inclination of this large arclet alone.

Accordingly, from the above sample, 8 multi-slit masks were constructed each containing between about 5 and 15 arclet targets which allowed for some duplication between the two runs. In addition to the main targets, spectra were obtained for several other objects, for instance where a part of the slit happened to fall on a second object near the main one or where a suitable arclet candidate was not available. These 'serendipitous' objects are at present included with the main sample but flagged as such on the following diagrams. In summary therefore, the spectroscopic sample consisted of 61 targets of which 37 were bona fide arclet candidates. The colour magnitude diagram for our targets is presented in Figure 2, superimposed on that for the whole cluster field. Our magnitude and colour limits can clearly be seen as can the similar (B-I) colours of several of the multiple image systems. The observations were completed during May/June 1995 and June 1996 under mixed conditions. A total of 7 masks were exposed as shown in Table 1.

The data were reduced using the LEXT package (Colless et al. 1990, Allington-Smith et al. 1994). Frames were debiassed and median combined taking into account small $\left(\sim 0.3\right.$ pixel $\left.h r^{-1}\right)$ shifts due to instrument flexure. Dispersed tungsten flat fields were used to correct for alongslit variations in illumination and pixel to pixel sensitivity changes with wavelength. Third order polynomial fits to $\mathrm{CuAr}$ arc lines provided wavelength calibration to a r.m.s. residual of $\sim 2.0 \pm 0.1 \AA$. Sky subtraction was performed in two steps. Firstly we fit the summed slit profiles, excluding the object rows and edge effects. The deviation from this overall profile was then fit column by column to remove wavelength variations in the profile. Finally the object rows were extracted with a Gaussian weighting to optimize signal to noise (except for cases where the target was partially

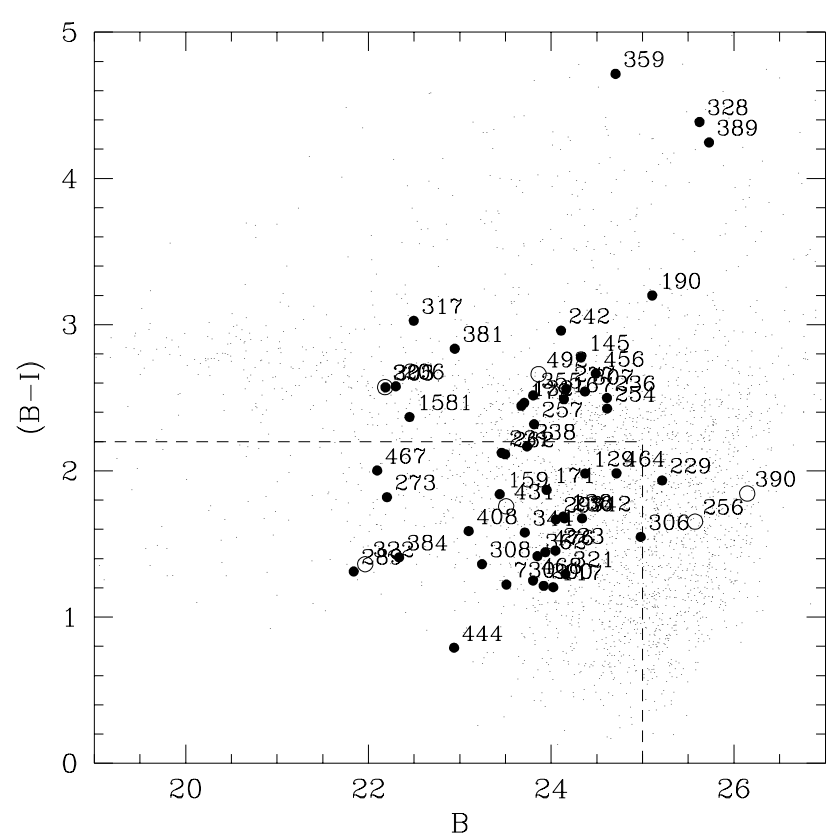

Figure 2. The colour magnitude diagram for the arc sample superimposed on that for the whole cluster field. Open circles denote non-sample objects which were not obviously cluster members and the dashed lines show our magnitude and colour criteria. Objects outside this region were selected primarily by morphology or are serendipitous. Note the similar $(B-I)$ colours of the multiple image systems $(384,468)$ and $(359,328,389)$.

merged with another object, e.g. \#273). Redshifts were determined independently by two observers (RSE and TMDE) using Karl Glazebrook's REDSHIFT utility and are summarized in Table 2. We assigned a quality flag $q$ to each spectrum according to the reliability of the redshift determination. Redshifts determined from more than one strong line, or a single line and continuum with good signal to noise $(\mathrm{S} / \mathrm{N})$ were assigned $q=1$, designated 'certain / probable'. Those determined from one or more noisy lines were assigned $q=2$, designated 'possible', and those for which no redshift could be obtained were given $q=3$.

Of the 37 arclet candidates for which slits were cut, 7 failed to yield spectra of adequate signal/noise (never reaching $S / N_{\text {cont }} \simeq 3$ ). There are several reasons for this. Firstly, a major limiting factor at these faint limits is the effectiveness of sky subtraction. This is largely influenced by the surface brightness of the target compared to that of the night sky (nominally $\mu_{R, s k y} \simeq 20.4 \mathrm{mag} \mathrm{arcsec}^{-2}$ at La Palma in dark time.) Figure 3 shows the success rate of our survey with both integrated $R$ magnitude and surface brightness, $\mu_{R}$. It is evident that our sample is strictly limited at the $\mu_{R}=24$ level and that our percentage success rate for determining firm redshifts decreases as the mean surface brightness falls $\left(100 \%, 54 \% \& 28 \%\right.$ in the three bins from $\mu_{R}=22$ to $\mu_{R}=24$ respectively.) 6 of the 7 low $\mathrm{S} / \mathrm{N}$ arclets have surface brightnesses $\mu_{R} \geq 23.2$ ( $\leq 7 \%$ of the night sky) and integrated magnitudes of $B \geq 24.0$ ( $R>22.0$ ), the faintest limit attained by previous LDSS- 2 surveys. In addition, in all but one case, less than 8 arcseconds of sky was available for sky subtraction due either to contamination by a 
Figure 1. HST WFPC2 image of the cluster Abell 2218 in F702W showing the sample of objects selected for spectroscopy (listed in table 2). Those for which we have obtained good redshifts are marked with filled labels.

Table 1. Spectroscopic Observations. The LDSS-2 medium blue grism was used for all masks except mask 1 for which the medium red grism was used. Both grisms give a resolution of $\sim 12 \AA$. During the 1995 run a thinned Tektronix 1024 ${ }^{2}$ CCD (TEK-1) was the detector, giving 0.59 arcsec pix ${ }^{-1} \& 5.3 \AA$ pix -1 while a new Loral $2048^{2}$ CCD (LOR-1) became available for the 1996 run, with a scale of 0.37 arcsec pix ${ }^{-1} \& 3.3 \AA$ pix $^{-1}$. Final stacked on-source exposure times were as follows: mask $1-19.8 \mathrm{ksec}$, mask $2-19.1 \mathrm{ksec}$, mask 4 - 10.8ksec, mask $730-32.4 \mathrm{ksec}$, mask $444-45.5 \mathrm{ksec}$, mask323A - 10.0ksec, mask323B - 7.2ksec. NOTES: (1) Identification numbers follow Le Borgne, 1992. (2) Duplicate masks. These contained the same objects except that the bright target \#323 on mask 323A was replaced on mask 323B by fainter targets to increase the number of spectra obtained. (3) Numbers in parentheses denote exposures in poor conditions.

\begin{tabular}{|c|c|c|c|c|}
\hline \# Mask & Targets $^{1}$ & Dates & Total Exposure $(\mathrm{ksec})^{3}$ & Seeing $(\operatorname{arcsec})$ \\
\hline \multirow[t]{2}{*}{1} & $138,117,231,321,456,355,308,236,408$ & 1995 May 27 & 10.8 & 1.0 \\
\hline & " & 1995 May 29 & 9.0 & $1.0-1.5$ \\
\hline \multirow[t]{2}{*}{2} & $129,171,229,328,384,468,492$ & 1995 May 28 & 5.4 & $1-2$ \\
\hline & " & 1995 May 30 & 11.9 & 1.0 \\
\hline 4 & $238,273,384,467$ & 1995 June 1 & 10.8 & $0.7-1.0$ \\
\hline \multirow[t]{3}{*}{730} & $730,158,167,179,230,254,257,342,464,476,461$ & 1996 June 18 & 14.4 & 1.1 \\
\hline & $"$ & 1996 June 19 & $7.2(3.6)$ & 1.0 \\
\hline & “ & 1996 June 20 & 7.2 & 1.0 \\
\hline \multirow[t]{3}{*}{444} & $444,117,145,236,242,256,262,297,306,307,308,344,381,431$ & 1996 June 16 & $7.5(7.5)$ & $0.8-1.3$ \\
\hline & (") & 1996 June 17 & 15.0 & $0.8-1.0$ \\
\hline & “ & 1996 June 21 & 18.0 & $0.9-1.1$ \\
\hline $323 \mathrm{~A}^{2}$ & $323,132,159,167,190,200,205,223,288,295,317,321,389$ & 1996 June 15 & 10.0 & $1.0-1.5$ \\
\hline $323 \mathrm{~B}^{2}$ & $277,132,159,167,190,200,205,223,288,295,257,321,389$ & 1996 June 20 & 7.2 & 0.9 \\
\hline
\end{tabular}

second galaxy in the slit or simply the length of the slit. Finally, it is possible that misalignment of the slits from their required positions could have led to low signal to noise in some spectra. To investigate this, we took all the targets with $22<R<23$ and measured the average count level in each spectrum in $\mathrm{a} \sim 1000 \AA$ wide region spanning the position of the $\mathrm{R}$ filter $(6500 \AA)$. If most slits were aligned well with the target objects, there should be a correlation between the count level in the spectra and $\mathrm{R}$ magnitude. We performed this test for 15 arclets and indeed found a linear correlation of spectral counts with $10^{-0.4 R}$ when three outliers were discarded. At least one of the outliers could be explained by poor slit orientation, suggesting that misalignment of the slits with object positions was not a significant cause of light loss in the majority of cases.

Several spectra show good continuum signal to noise, but no recognizable features and it is reasonable to ask what redshift ranges are implied by the non-detection of features in these spectra. Following Colless et al. (1990), we can use those spectra where [OII] emission was detected to calculate our sensitivity to detection of emission features. Given a continuum $\mathrm{S} / \mathrm{N}$ value one may obtain a limit on the minimum equivalent width of emission which could result in a detection for a particular spectrum. As the continuum $\mathrm{S} / \mathrm{N}$ increases, we should become increasingly sensitive to lower equivalent widths of emission. Figure 4 shows a plot of the continuum $\mathrm{S} / \mathrm{N}$ adjacent to [OII] versus the observed equivalent width of [OII] for the 15 arclets from which [OII] emission was detected. For an unresolved line, continuum $\mathrm{S} / \mathrm{N}\left(S / N_{\text {cont }}\right)$, line equivalent width $\left(W_{\lambda, o b s}\right)$ and line $\mathrm{S} / \mathrm{N}\left(S / N_{\text {line }}\right)$ can be related to each other, via simple assumptions, through the relation $S / N_{\text {cont }} W_{\lambda, \text { obs }} \approx$ $\sqrt{2 \pi} \sigma_{\text {line }} S / N_{\text {line }}$. (We assume the line profile is a Gaussian of sigma $\sigma_{\text {line }} \simeq 0.425 F W H M$. For our $\sim 12 \AA$ resolution, this gives $\sigma_{\text {line }} \simeq 5.1$.) Plotting this relation on the diagram shows that our detection limit corresponds to a value of $S / N_{\text {line }} \simeq 5$.

We now proceed to use this detection limit to put bounds on the possible redshifts of those $(q=3)$ arclets with good S/N but no visible features. Given some signal to noise ratio for a piece of continuum, one may calculate using the relation of Figure 4 the corresponding minimum equivalent width of emission which could have been detected there. Combining this limit with the distribution of [OII] equivalent widths seen in field galaxies from other surveys, we may derive a probability that there could be [OII] emission at this point without it having been detected. The presence of [OII] at this point defines a redshift and so this translates into a probability $p_{S / N}(z)$ that the galaxy could have this particular redshift without any [OII] being detected. Thus one may derive a $p_{S / N}(z)$ value for each point on the spectrum once its $S / N_{\text {cont }}$ has been measured. At these faint limits, the signal to noise in the red becomes prohibitive when one encounters sky emission lines, and so we have chosen to apply this measurement to 7 windows between sky

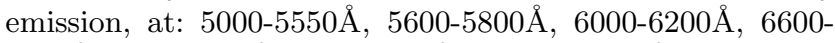
$6800 \AA, 7100-7200 \AA, 7600-7700 \AA$, \& 8100-8270 . These correspond to redshift windows for [OII] of $z=0.34-0.49,0.50$ 0.56,0.61-0.66,0.77-0.82,0.91-0.93,1.04-1.07 \& 1.17-1.22. Figure 5 shows 3 examples where $p_{S / N}(z)$ has been calculated. To obtain a redshift constraint, we define a threshold for $p_{S / N}(z)$. We can then use the point at which the interpolated $p_{S / N}(z)$ curve rises above this threshold as a limit on the redshift. Here we choose a value of $p_{S / N}(z)=0.5$ and 

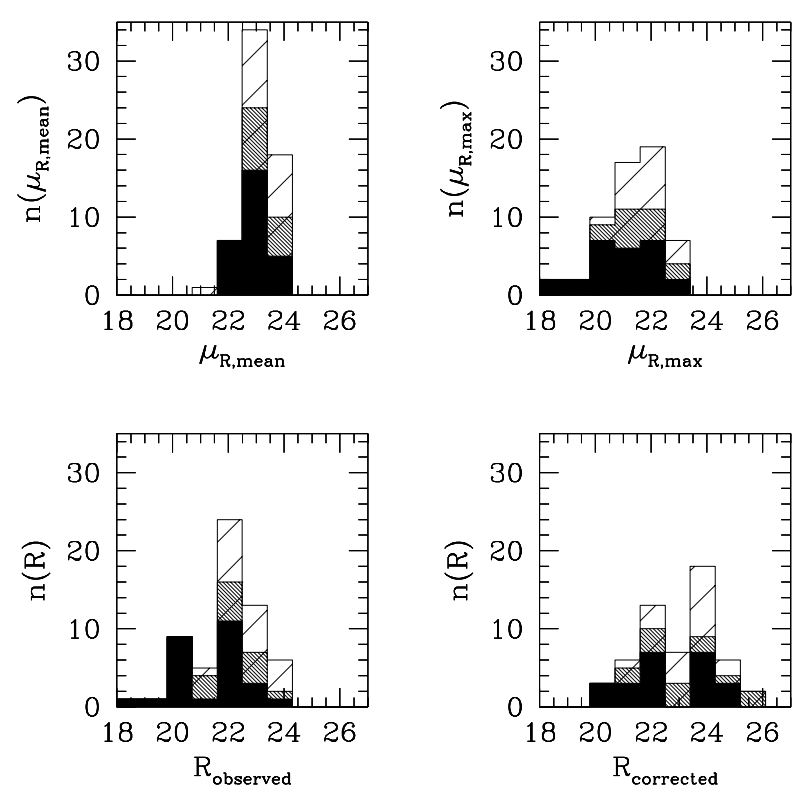

Figure 3. Redshift completeness of the sample versus mean surface brightness $\left(\mu_{R, \text { mean }}\right)$, peak surface brightness $\left(\mu_{R, \max }\right)$, observed magnitude $\left(R_{\text {observed }}\right)$ and magnitude corrected for lensing amplification $\left(R_{\text {corrected }}\right)$. Light, heavy and solid shading represent arclets for which redshifts were undetermined, tentative and firm respectively. It is evident that our success rate for obtaining good redshifts falls as both the surface brightness and total observed magnitude fall. However, the bottom right panel indicates that, with the help of lensing amplification, we have obtained redshifts down to $R \simeq 25.5$.

quote the redshift limits obtained for these $q=3$ spectra in Table 2 .

There are several caveats to this process. Firstly, the value $p_{S / N}(z)$ is only valid within the sky window where the signal to noise was measured. It is possible that a significant equivalent width of [OII] could remain undetected between these windows if the continuum signal to noise in the sky band was low enough. Secondly, we assume here that the distribution of observed equivalent widths corresponds to those of the LDSS-2 deep redshift survey (Colless et al. 1990). We note that this produces a conservative estimate since our survey pushes significantly deeper in magnitude and redshift thus making it likely that the true [OII] equivalent width distribution of our sample contains more large equivalent widths than the one we have used. This would lead to an over estimate of $p_{S / N}(z)$ for any given continuum signal to noise. Finally, we point out that this method will produce erroneous results if our sample does not correspond to the general field in its distribution of equivalent widths. To summarize, we have used our detection limit for emission lines to derive redshift limits for those spectra which show good continuum signal to noise but no features.

Figure 7 shows how our redshift completeness varies with the target colours. It can be readily seen that more redshifts were obtained for blue objects than red. This probably reflects the greater strength of [OII] emission in the blue objects as hypothesised in our selection method. This is confirmed in Figure 6 which shows the correlation be-

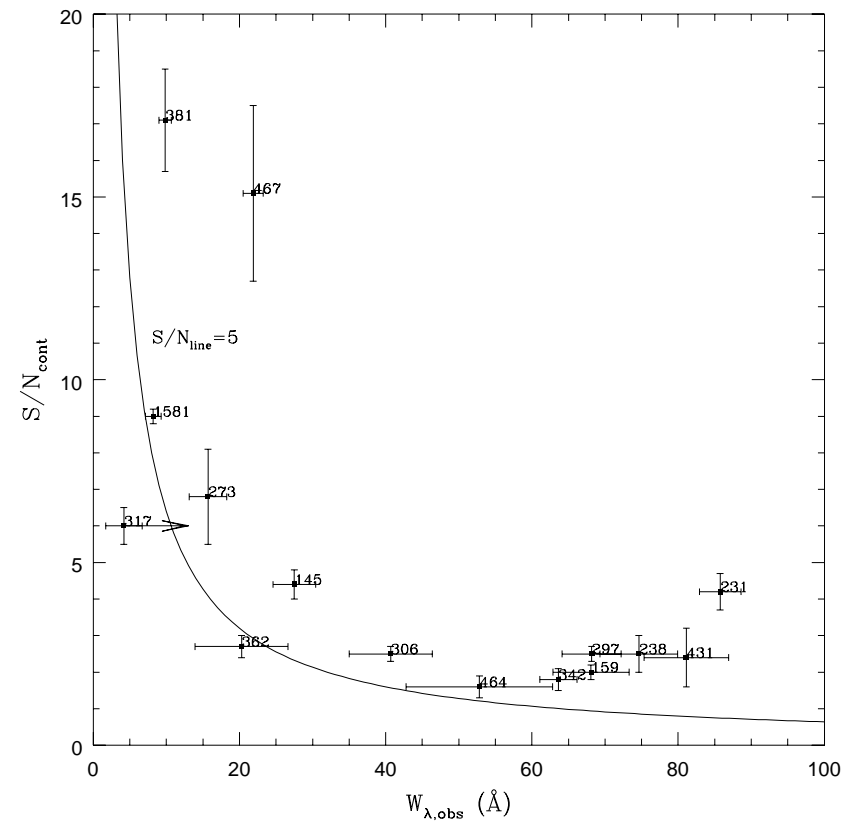

Figure 4. Continuum signal to noise ratio versus observed [OII] equivalent width for the arclets with $q=1$ (after Colless et al., 1990). The curve shows a detection limit of $S / N_{\text {line }}=5$. Due to the proximity of [OII] to the $\lambda 5577$ sky emission line in \#317, the equivalent width shown for this object is only a lower limit. Also shown is one candidate with $q=2$ (\#362) which falls just below the line.

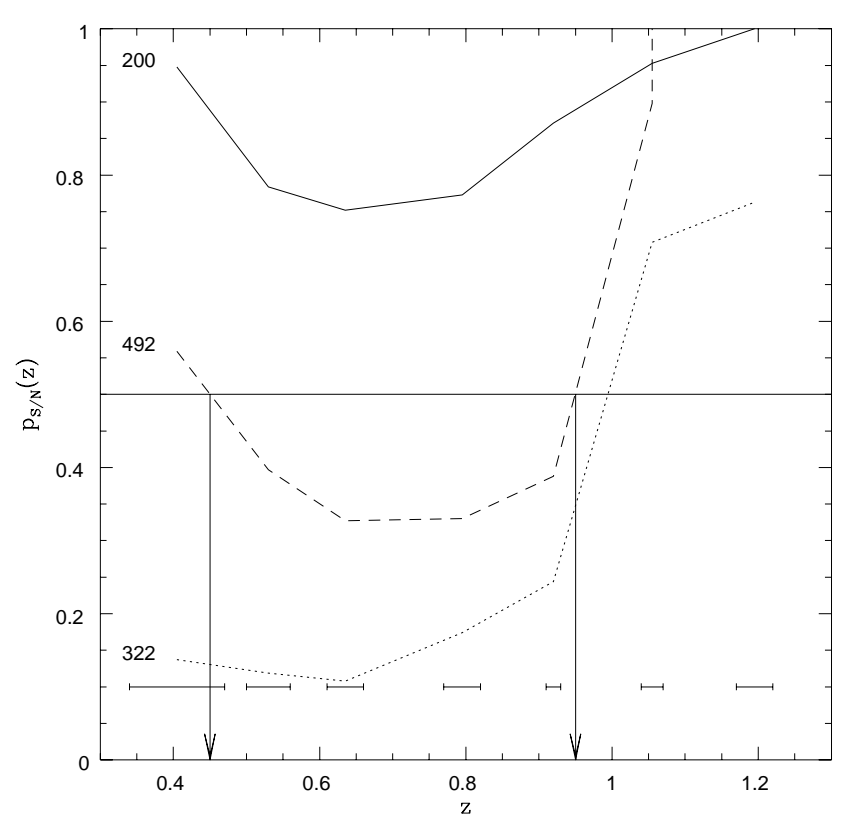

Figure 5. Examples of the ' $p(z)$ ' curves derived from the signal to noise arguments given in $\S 2$. The vertical arrows show how the redshift constraint is defined. The three examples show one case giving a single lower bound (\#322), one giving both upper and lower limits (\#492) and one giving no bound at all (\#200). The horizontal bars at the bottom of the plot show the size of the redshift windows within which the probability value applies. 


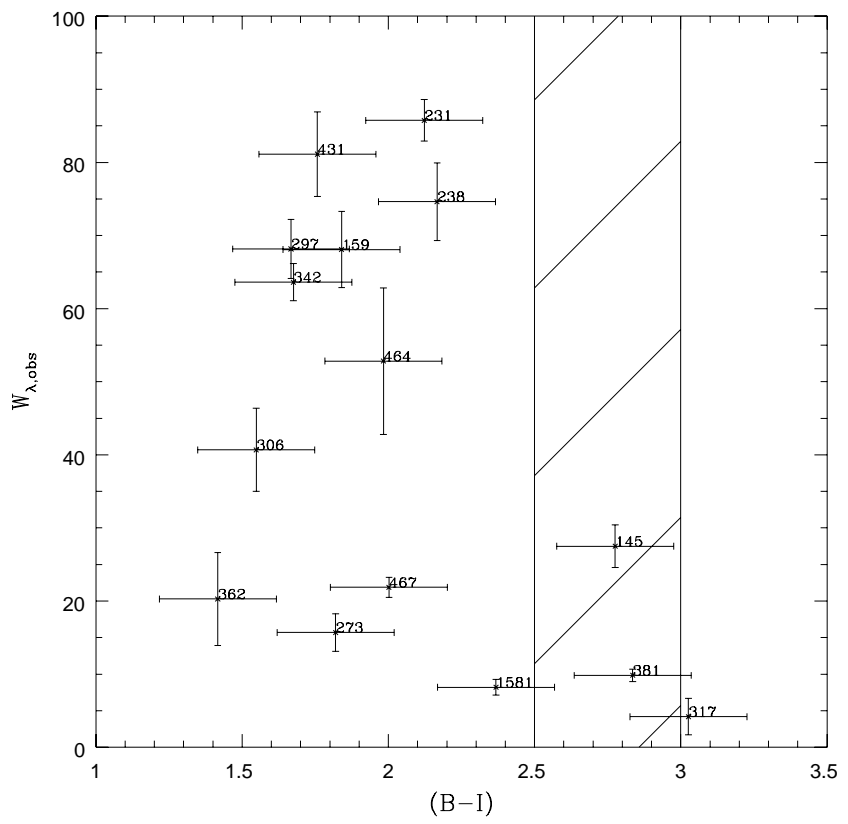

Figure 6. The correlation between observed equivalent width and $(B-I)$ colour for those targets where [OII] was detected. The shaded area encloses the region occupied by the E/S0 sequence of the cluster. [OII] emission was detected in only one cluster member (\#342) and in fact this object is well outside the cluster sequence. It is thus clear that the blue colour selection criterion proved a good predictor of the presence of [OII] .

tween equivalent width in $[\mathrm{OII}]$ and $(B-I)$ colour. Here, the shaded region marks the cluster sequence colour. [OII] emission was detected for only one cluster galaxy which, in fact, lies well outside the cluster sequence. There is a clear anticorrelation between $(B-I)$ colour and equivalent width such that the reddest galaxies have little or no [OII] emission. This confirms the results seen by Colless et al. (1990) and Glazebrook et al. (1995). We conclude that our colour selection was a useful way to increase the likelihood of detecting [OII] emission (and thus obtaining a redshift), as well as a method for selecting non-cluster objects.

Of the 37 arclet targets, we were able to obtain a total of 32 redshifts, 18 of which were of good quality $(q=1)$ and were behind the cluster $\left(z_{s}>0.2\right)$. Figures $8,9,10$ and 11, show the extracted spectra, 2-dimensional spectra and zooms of the arclets, while the redshift catalogue is presented in Table 2 .

Several of the spectra merit individual discussion especially with regard to their redshift determinations. First we deal with the multiple images and follow with those that are singly imaged.

$\mathbf{2 8 9}(\mathrm{R}=20.5, \mathrm{z}=1.034) \& \mathbf{3 5 9},(\mathrm{R}=20.3, \mathrm{z}=0.702)$

The spectra for these giant arcs are discussed by Pelló et al. (1992).

$328(\mathrm{R}=22.0, \mathrm{z}=0.702) \& \mathbf{3 8 9}(\mathrm{R}=21.5, \mathrm{z}=0.702)$ Both part of the \#359 multiple system showing weak features corresponding to the \#359 redshift of $z=0.702$. \#389 appears seen through the disc of the spiral galaxy \#373 and consequently the features are so weak that we assign $q=2$. $273(\mathrm{R}=21.9, \mathrm{z}=0.800)$ The spectrum of this arc is heavily

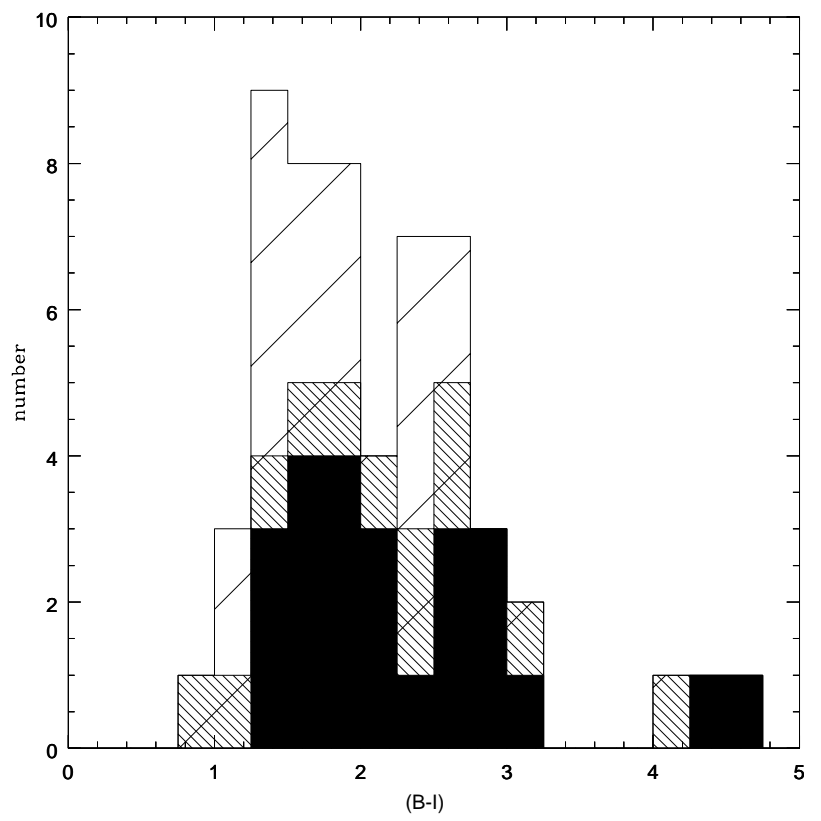

Figure 7. The $(B-I)$ distribution of the spectroscopic sample. As before, light, heavy and solid shading represent undetermined, tentative and confirmed redshifts respectively.

contaminated with light from the nearby elliptical \#268 (see Figure 9). However, as may be seen from the 2-d spectrum, the emission line is very prominent at the lower edge of the \#268 continuum. Only the 3 rows containing the emission line were extracted and no Gaussian weighting was used.

$384(\mathrm{R}=21.2, \mathrm{z}=2.515)$ The spectrum of this spectacular arc has already been discussed in a recent letter (Ebbels et al. 1996.) Many rest frame ultra-violet metal lines are seen, along with Lyman- $\alpha$ in absorption at $z=2.515$.

$444(\mathrm{R}=22.7, \mathrm{z}=1.030)$ The spectrum has good continuum signal to noise and is extremely blue. We base our identification on the FeII doublet at $\lambda 2587$ and $\lambda 2600$. This is in good agreement with the predicted redshift of $z=1.1 \pm 0.1$. However this remains a tentative $(q=2)$ identification.

$145(\mathrm{R}=22.1, \mathrm{z}=0.628)[\mathrm{OII}]$ is seen strongly in this spectrum, along with some other more tentative features.

$158(\mathrm{z}=0.721)$ This spectrum could be a blend of 2 sources as may be inferred from Figure 8 . We identify several features (principally [OII]) at a redshift of $z=0.723$ while other features, including the continuum, are more consistent with a redshift of $z=0.167$. We suggest that most of the continuum comes from a bright cluster member while the fainter (by $0.7 \mathrm{mag})$, diffuse object is a background galaxy $(\mathrm{B}=22.5$, henceforth denoted \#1581) and produces the [OII] emission. Unfortunately the ground based seeing precludes any spatial identification of the emission line with one particular component.

$159(\mathrm{R}=21.9, \mathrm{z}=0.564)[\mathrm{OII}]$ emission dominates this spectrum which also includes several absorption features.

$205(\mathrm{R}=20.3, \mathrm{z}=0.693)$ This spectrum has good signal to noise with both absorption and emission features around the Balmer break visible.

231 ( $R=22.0, \mathrm{z}=0.563)$ The ground-based seeing causes this spectrum to be a blend of 231 and compact component. 
These have $\mathrm{R}$ magnitudes of 22.0 and 23.4 respectively, so that no more than $21 \%$ of the light can be contributed by the compact component and it is unlikely that the emission line arises from it alone.

$238(\mathrm{R}=21.9, \mathrm{z}=0.635)$ A single emission feature identified with $[\mathrm{OII}]$ dominates this spectrum.

$242(\mathrm{R}=21.8, \mathrm{z}=654)$ Again, $[\mathrm{OII}]$ emission is seen along with several absorption features at lower signal to noise.

$297(\mathrm{R}=22.4, \mathrm{z}=0.450)$ [OII] emission is very strong here and is accompanied by tentative identifications of the $H \beta /[\mathrm{OIII}]$ triplet.

$306(\mathrm{R}=23.7, \mathrm{z}=0.450)[\mathrm{OII}]$ emission along with several other more tentative identifications lead to the redshift for this arclet.

$317(\mathrm{R}=20.1, \mathrm{z}=0.474)$ The [OII] emission here falls very close to the $\lambda 5577$ sky line and so we can determine a lower limit only on its equivalent width. However, several other features confirm the redshift.

$381(\mathrm{R}=20.5, \mathrm{z}=0.521)$ This spectrum, which has one of the best signal to noise ratios of all those obtained, exhibits many features from MgII absorption to $H \beta$.

$431(\mathrm{~B}=23.5, \mathrm{z}=0.675)$ Despite its proximity to the $\lambda 6300$ night sky emission line, the [OII] emission in this arclet is strong and unmistakable on the 2-d spectrum.

$456(\mathrm{R}=22.2, \mathrm{z}=0.538)$ Several absorption features including the $\mathrm{K} / \mathrm{H}$ doublet are visible with good enough signal to noise to obtain a redshift for this arclet.

$464(\mathrm{R}=22.9, \mathrm{z}=0.476)$ A strong emission feature identified with $[\mathrm{OII}]$ plus a possible $\mathrm{G}$ absorption band lead to a redshift for this arclet.

$467(\mathrm{R}=20.5, \mathrm{z}=0.475)$ Excellent signal to noise provides a sure redshift for this arclet, with up to 14 identifications from [OII] emission to $\lambda 5268$ absorption.

We also mention the object \#323 which, being very elongated but at an angle $\gtrsim 45^{\circ}$ from the shear direction, would cause problems for the model were it at a redshift much higher than the cluster. Identification of several absorption features in this spectrum reveal it to be a cluster member at a redshift of $z=0.179$, thus posing no threat to the model. Finally, we note that of our new redshifts, 7 arclets are found in three redshift structures. The sensitivity to small-scale redshift clustering is an artifact of the small angular extent of the survey and is a factor affecting all small-area surveys (including the photometric redshift surveys in the Hubble Deep Field, see §5). Thus, gaining a statistically-reliable view of the field redshift distribution will require inversion through a number of lenses.

The 18 new redshifts bring the total number of arclets with good redshifts in Abell 2218 to 20 - making it the largest number for any lensing cluster to date. Table 2 lists the results in order quality with multiple image systems appearing in the top 2 sections. The following analysis of the inversion method and our conclusions are based solely on the best quality $(q=1)$ arclets, though we expand the sample to include the $q=2$ arclets where appropriate. For completeness we also list the $q=3$ arclets and their redshift limits from the signal to noise argument above in order to give an indication of the sample which might be reached with a $10 \mathrm{~m}$ class telescope.

\section{COMPARISON WITH LENSING PREDICTIONS}

We now compare the redshifts obtained with those predicted by the lens inversion method outlined in KESCS.

The arcs whose redshifts can be predicted fall into two classes: multiple images and single images. The redshift predictions for the multiple images have much smaller uncertainties than those for singly-imaged sources because of the larger number of constraints implied when several images of the same source are taken together. It is therefore particularly important to prove that that the inversion works for these sources. However, in the case of a singly-imaged arclet, the uncertainty in the redshift prediction is more typical of that expected for the larger fainter population of arclets addressed by KESCS. The uncertainty here depends on the redshift, the size of the local shear and the unknown intrinsic shape of the source. The average uncertainties listed in KESCS were $\delta z / z=22 \%$ for multiple images and $\delta z / z=30 \%$ for single images.

In order to understand what level of agreement we expect between our inverted redshifts and measured spectroscopic ones, it is useful to consider the method by which the inverted redshifts are obtained. For any particular singly imaged arclet at any point on our image, our mass model can predict magnitude and direction of the shear induced by the lens. The magnitude of this shear depends on the redshift of the arclet and increases from a minimum at the cluster redshift to an absolute maximum as the arclet redshift approaches infinity (This limit is actually replaced by a redshift of $\sim 4$ in our model). The observed shape of the arclet is a combination of its intrinsic shape and this redshift dependent distortion. Conversely, knowing the observed shape, and given a redshift, one can predict the intrinsic shape using the calculated shear. As we move the arclet in redshift from the distance of the cluster out to infinity, its predicted intrinsic shape will change, eventually tending to a constant shape at large redshift.

We may represent the shape of any object on a plot of ellipticity vectors $\bar{\tau}$ as shown in Figure 12 . Using the notation of KESCS, $\bar{\tau}=\tau e^{2 i \theta}$ and $\tau=\left(a^{2}-b^{2}\right) /(2 a b)$ with $a$ and $b$ the semi-major axes of the equivalent ellipse. The vectors may be plotted with respect to the axes of the CCD (large plots) or with respect to the axis of local shear for each arclet (insets). In these local shear axes, the lensing transformation conserves the y component of the ellipticity, $\tau_{y}$ and thus the process of moving the arclet in redshift simply corresponds to moving its point in the $-x$ direction across the $\bar{\tau}$-diagram. As we move the arclet out in redshift and thus to the left in $\tau_{x}$ across the plot, we can ask what is the likelihood that any given galaxy in the field would have the shape defined by this point on the $\bar{\tau}$ diagram. This likelihood will correspond to the value of the ellipticity distribution of field galaxies, $p(\bar{\tau})$ (represented by the contours on the plots) at that point. We can therefore define a redshift probability function $p(z)$ for each arclet, corresponding to the likelihood that its intrinsic shape for any redshift would be found in the general population of field galaxies. The $p(\bar{\tau})$ distribution peaks at an ellipticity of $\tau=0$ and has circular symmetry. Thus the maximum in our probability, $p\left(\tau_{x}\right)$ or correspondingly, $p(z)$ will occur when $\tau_{x}=0$. We therefore assign a most likely redshift $z_{o p t}$ to each arclet defined by 
Table 2. Arclet Spectroscopy in Abell 2218. We list first the multiple image systems, followed by the singly imaged ones. Only arclets with $z_{s} \geq 0.2$ are listed, except in the case of \#323 (see text). The arclets for which no redshift prediction was possible were added to the sample at points on the masks where suitable bright arclets with inverted redshifts were unavailable. COLUMN NOTES: (1) $R_{\text {corr }}$ calculated assuming $z=z_{\text {opt }}$. (2) Quality (q): 1=certain/probable, 2=possible, 3=limit only (3) From WFPC-2 F702W image transformed to R as detailed in KESCS. Estimated error 0.2 mag. (4) For those objects with undetermined redshifts, we quote limits on the redshift derived from the argument in §2. COMMENTS: (1) Class III; No maximum in $p(z): z_{\text {opt }} \rightarrow \infty$ (2) Class I; No maximum in $p(z): z_{\text {opt }} \leq 0.2$ (3) Serendipitous object (4) Arclet off WF frame, photometry quoted in B band (5) Redshifts determined by Pelló et al., 1992 .

\begin{tabular}{|c|c|c|c|c|c|c|c|c|c|c|c|}
\hline$\#$ & $\mu_{R}$ & $\mathrm{R}^{(3)}$ & $R_{\text {corr }}^{(1)}$ & $z_{\text {spec }}^{(4)}$ & $z_{-}$ & $z_{o p t}$ & $z_{+}$ & $\delta_{z} / z_{o p t}$ & quality $(\mathrm{q})^{(2)}$ & identified features & comment \\
\hline 289 & 23.1 & 20.5 & 22.3 & 1.034 & & 1.034 & & & 1 & {$[O I I]$} & 5 \\
\hline 359 & 22.7 & 20.3 & 24.9 & 0.702 & & 0.702 & & & 1 & $K, H, H \gamma$ & 5 \\
\hline 328 & 22.6 & 22.0 & 24.9 & 0.702 & & 0.702 & & 0.00 & 1 & $K ?, H, 4000$ & \\
\hline 389 & 22.8 & 21.5 & 24.9 & 0.702 & & 0.702 & & 0.00 & 2 & $K ?, H ?$ & \\
\hline 384 & 23.4 & 21.2 & 24.1 & 2.515 & 2.6 & 2.8 & 3.3 & -0.10 & 1 & $L y-\alpha, S i I I, O I, C I I, S I V, C I V$ & \\
\hline 444 & 23.9 & 22.7 & 25.7 & 1.030 & 1.0 & 1.1 & 1.2 & -0.06 & 2 & FeII? & \\
\hline 145 & 23.3 & 22.1 & 22.3 & 0.628 & 0.5 & 0.9 & 2.9 & -0.3 & 1 & {$[O I I], K ?, H ?, H \delta ?$} & \\
\hline 1581 & & 22.5 & 23.0 & 0.721 & 0.3 & 0.6 & 1.4 & 0.20 & 1 & $M g I I ?,[O I I]$ & 4 \\
\hline 159 & 22.7 & 21.9 & 21.9 & 0.564 & 0.2 & 0.2 & 0.2 & - & 1 & {$[O I I], H \theta ?, H \eta ?, H \zeta ?, H ?,[O I I I] ?$} & 2 \\
\hline 205 & 22.4 & 20.3 & 21.1 & 0.693 & 0.2 & 0.3 & 0.4 & 1.3 & 1 & {$[O I I], H \theta, H \eta, H \zeta, K, H, H \delta$} & \\
\hline 231 & 23.1 & 22.0 & 22.2 & 0.563 & 0.3 & 0.4 & 0.6 & 0.4 & 1 & {$[O I I], K ?, H ?, 4000$} & \\
\hline 238 & 23.5 & 21.9 & 23.9 & 0.635 & 0.8 & 1.2 & 1.6 & -0.5 & 1 & {$[O I I], H ?, 4000 ?$} & \\
\hline 242 & 23.2 & 21.8 & 21.8 & 0.654 & 0.2 & 0.2 & 0.2 & - & 1 & {$[O I I], H \zeta ?, K ?, H ?, H \delta ?$} & 2 \\
\hline 273 & 22.2 & 21.9 & 23.8 & 0.800 & 0.5 & 0.6 & 0.7 & 0.33 & 1 & {$[O I I]$} & \\
\hline 297 & 23.7 & 22.4 & 23.8 & 0.450 & 0.5 & 0.6 & 0.7 & -0.3 & 1 & {$[O I I], H \delta ?, H \beta,[O I I I]$} & \\
\hline 306 & 22.8 & 23.7 & 24.2 & 0.450 & - & - & - & - & 1 & {$[O I I], H \zeta, H ?, G ?, H \beta,[O I I I]$} & 1 \\
\hline 317 & 22.8 & 20.1 & 20.6 & 0.474 & 0.2 & 0.3 & 0.4 & 0.6 & 1 & {$[O I I], H \eta, H \zeta, K, H, 4000, H \delta$} & \\
\hline 381 & 22.7 & 20.5 & 21.0 & 0.521 & 0.3 & 0.4 & 0.5 & 0.3 & 1 & $M g I I ?,[O I I], H \theta, H \eta, H \zeta, H \delta, G$ & \\
\hline 431 & & 23.5 & & 0.675 & - & - & - & - & 1 & $M g I I ?, M g I ?,[O I I]$ & 4 \\
\hline 456 & 23.4 & 22.2 & 24.5 & 0.538 & 0.4 & 0.6 & 0.8 & -0.1 & 1 & {$[O I I] ?, H \zeta ?, K, H, 4000, G ?$} & \\
\hline 464 & 23.3 & 22.9 & 24.8 & 0.476 & 0.9 & 1.1 & 1.3 & -0.6 & 1 & {$[O I I], G ?$} & \\
\hline 467 & 22.1 & 20.5 & 21.7 & 0.475 & 0.4 & 0.4 & 0.5 & 0.2 & 1 & $\begin{array}{l}{[O I I], H \theta, H \eta, H \zeta, K, H, 4000, H \delta} \\
G ?, H \beta ?,[O I I I] ?, M g b ?, 5268 ?\end{array}$ & \\
\hline 132 & & 23.7 & 23.7 & 0.703 & 0.2 & 0.2 & 0.2 & - & 2 & $M g I ?, M g I I ?$ & 2,4 \\
\hline 190 & 23.4 & 22.1 & 22.1 & 0.708 & 0.2 & 0.2 & 0.2 & - & 2 & {$[O I I] ?$} & 2 \\
\hline 229 & 23.8 & 23.8 & 24.8 & 0.830 & 0.8 & 1.0 & 1.2 & -0.2 & 2 & $M g I ?, M g I I ?$ & \\
\hline 236 & 23.3 & 22.4 & 23.1 & 0.570 & 0.4 & 0.5 & 0.6 & 0.1 & 2 & $H \theta ?, H \eta, K ?, H \gamma ?$ & \\
\hline 262 & 23.7 & 21.8 & 21.8 & 0.596 & 0.2 & 0.2 & 0.2 & - & 2 & $K ?, H ?, 4000 ?, H \delta ?, H \beta ?$ & 2 \\
\hline 307 & 23.2 & 22.1 & 23.1 & 0.390 & 0.3 & 0.3 & 0.4 & 0.3 & 2 & $K ?, 4000 ?, G ?, M g b ?$ & \\
\hline 355 & 23.7 & 23.0 & 23.5 & 0.470 & 0.3 & 0.4 & 0.6 & 0.2 & 2 & $G ?$ & \\
\hline 362 & 23.9 & 22.6 & 25.8 & 0.532 & 0.5 & 1.1 & 2.0 & -0.5 & 2 & {$[O I I] ?$} & \\
\hline 117 & 23.1 & 23.0 & 23.4 & - & - & - & - & & 3 & & 1 \\
\hline 129 & 22.8 & 23.4 & 23.8 & - & 0.3 & 0.5 & 0.8 & & 3 & & \\
\hline 167 & 23.7 & 22.1 & 22.4 & $>0.44$ & - & - & - & & 3 & & 1 \\
\hline 171 & 23.3 & 22.3 & 23.0 & - & 0.4 & 0.6 & 0.8 & & 3 & & \\
\hline 179 & 23.4 & 21.9 & 22.1 & - & - & - & - & & 3 & & 1 \\
\hline 200 & 23.2 & 23.5 & 25.0 & - & 0.9 & 1.1 & 1.4 & & 3 & & \\
\hline 223 & 22.9 & 23.1 & 24.1 & $<0.53,>0.72$ & 0.6 & 0.7 & 0.9 & & 3 & & \\
\hline 230 & 23.3 & 22.6 & 24.0 & - & 0.4 & 0.5 & 0.6 & & 3 & & \\
\hline 254 & 23.9 & 22.0 & 23.9 & - & 0.4 & 0.5 & 0.7 & & 3 & & \\
\hline 256 & 23.2 & 23.9 & 24.1 & - & 0.2 & 0.4 & 0.8 & & 3 & & \\
\hline 257 & 23.2 & 21.9 & 22.7 & $>0.90$ & 0.3 & 0.5 & 0.8 & & 3 & & \\
\hline 277 & 23.3 & 22.4 & 23.4 & $>0.95$ & 0.3 & 0.4 & 0.5 & & 3 & & \\
\hline 308 & 23.4 & 22.2 & 23.4 & $>0.95$ & 0.5 & 0.6 & 0.8 & & 3 & & \\
\hline 322 & 21.3 & 21.1 & 21.1 & $>0.99$ & 0.2 & 0.2 & 0.2 & & 3 & & 2,3 \\
\hline 344 & 23.8 & 22.9 & 22.9 & $>0.92$ & 0.2 & 0.2 & 0.2 & & 3 & & 2 \\
\hline 390 & 23.7 & 24.1 & 24.1 & - & 0.2 & 0.2 & 0.2 & & 3 & & 2,3 \\
\hline 468 & 23.5 & 22.6 & 23.6 & $>0.81$ & 2.6 & 2.8 & 3.3 & & 3 & & \\
\hline 476 & 23.7 & 23.1 & 23.8 & $<0.45,>0.75$ & 0.3 & 0.3 & 0.4 & & 3 & & \\
\hline 492 & 22.8 & 21.6 & 21.7 & $<0.45,>0.95$ & 0.2 & 0.2 & 0.2 & & 3 & & 2 \\
\hline 730 & 23.7 & 22.8 & 25.1 & $>0.57$ & 1.0 & 1.1 & 1.2 & & 3 & & \\
\hline 323 & 22.7 & 20.1 & 20.1 & 0.179 & 0.2 & 0.2 & 0.2 & -0.1 & 2 & $K, 4000, M g b, N a D, H \alpha$ & \\
\hline
\end{tabular}


Figure 8. LDSS-2 spectra for arclets in Abell 2218, listed in order of identification number. All spectra with $q=1$ are shown, while arclets \#362, \#389, \& \#730 are included for interest. (Arc \#730 is shown with a rest frame wavelength scale corresponding to its predicted redshift.) See text for individual discussion. The spectra have been fluxed and smoothed to the effective resolution of LDSS-2, but are not corrected for atmospheric absorption.

Figure 9. LDSS-2 spectra for arclets in Abell 2218. (Continued)

the redshift which places it at $\tau_{x}=0$ on the diagram of Figure 12

This method divides the singly imaged arclet population into three classes, two of which preclude a determination of $z_{\text {opt }}$ (see also Figure 7 of KESCS). The first, class I, corresponds to those arclets with $\tau_{x}<0$. These cannot be moved to $\tau_{x}=0$ by placing them behind the cluster and must therefore be either cluster or foreground galaxies, or background galaxies which are not distorted enough to lie within a $45^{\circ}$ cone about the axis of local shear. Therefore no meaningful $z_{\text {opt }}$ may be determined for them. The second, class II arclets constitute those which may be moved to $\tau_{x}=0$ by placing them at a particular redshift behind the cluster and a determination of $z_{o p t}$ is possible for these arclets. Finally, particularly in areas away from the cluster core where the shear is weak, we have some arclets (class III) which although aligned closely with the shear axis, do not reach $\tau_{x}=0$ even at infinite redshift. Again, no meaningful $z_{\text {opt }}$ can be given for these arclets.

We must emphasize that the redshift prediction $z_{\text {opt }}$ only has physical meaning in a statistical sense. That is, while the shape corresponding to $z_{\text {opt }}$ may not in fact be the actual intrinsic shape of the object, the distribution of these shapes across the population of inverted objects must be drawn from the distribution of field galaxy shapes, $p(\bar{\tau})$. If this is true, then while the individual $z_{\text {opt }}$ values may not always correspond to the true redshift, the redshift properties of the population as a whole (such as $\langle z\rangle$ and shape of the $n(z)$ ) should be correct. Later we assess the manner in which our inverted shape distribution (Figure 12, left) samples the field galaxy $p(\bar{\tau})$ but first we discuss individual cases of inversion, starting with multiple images.

In KESCS' model, seven multiply imaged systems were used to constrain the mass model. These were identified using similarities in their HST morphologies, colours, surface brightnesses and on general assumptions about the lensing geometry. Only two of these (\#359 and 289) already had redshifts from the spectroscopic study of Pelló et al. (1992). Furthermore, none had actually been confirmed spectroscopically as multiple. Thus our redshift for \#328, a component of the 5-image \#359 system is a strong vindication of the mass model and the ability of HST to identify multiple images. The spectrum of \#389 is consistent with its being a third image of is system although highly contaminated by light from the obscuring cluster spiral \#373. Indeed, the identification of this as a 5-image system was the major motivation of KESCS to extend their model to include galaxy scale mass components. These 5 images could not be modeled using cluster scale mass components alone. Therefore, this confirmation reinforces our confidence in the mass model down to very small scales $(\sim 75 k p c)$. The \#384 system consists of one merging image pair (arc \#384) and its counter-image (arc \#468). Ebbels et al. (1996) confirmed

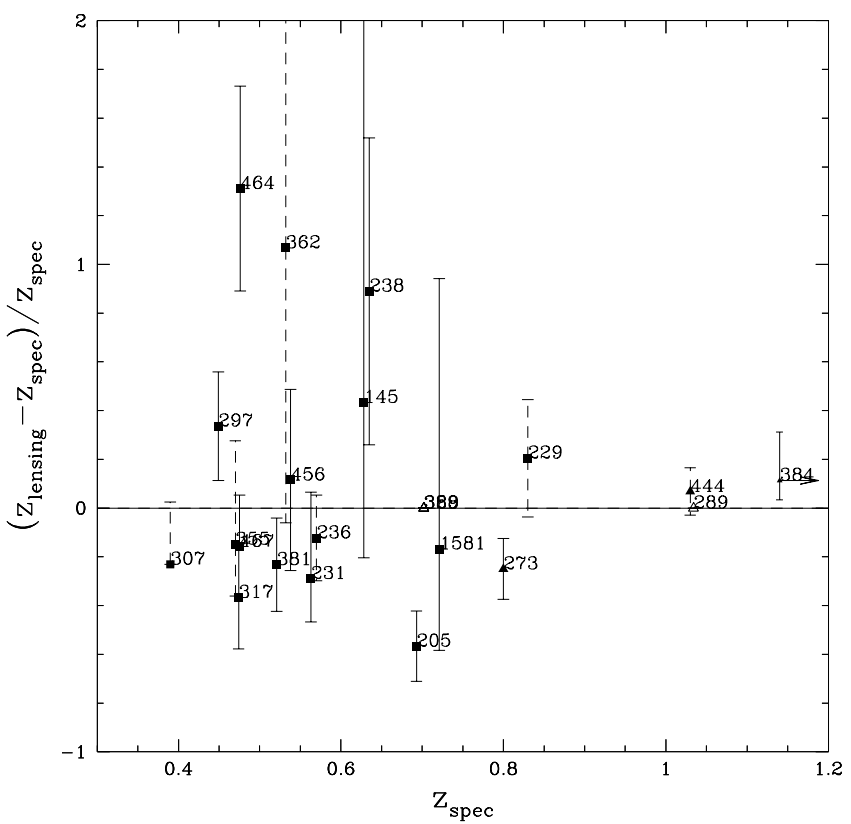

Figure 13. The fractional difference between $z_{\text {lensing }}$ and $z_{\text {spec }}$ for the A2218 sample. Single images are represented by squares and multiple images by triangles. The open triangles show multiple images that were used as constraints on the model. Objects with $q=1$ are shown with solid error bars, while those with $q=2$ have dashed error bars.

that both halves of the merging pair have the same redshift $(z=2.515)$ and also found the spectrum of \#468 to be compatible with this redshift, although its signal to noise is poor. Of particular significance, and also discussed briefly in Ebbels et al., is the fact that the spectroscopic redshift of this system is in excellent agreement with the lensing prediction: $z=2.8_{-0.3}^{+0.5}$ (KESCS). The redshift of this system is particularly important due to its proximity to a critical line, meaning that the predicted redshift is extremely sensitive to changes in the mass model. In summary, therefore, four images from two multiple systems are consistent with the earlier mass model and the most highly-magnified has a redshift within the expected uncertainties.

Turning to the singly-imaged arclets, Figure 13 shows the fractional difference between $z_{o p t}$ and $z_{\text {spec }}$ for the arcs and arclets where both a spectroscopic and inversion redshift above $z=0.2$ are available. Here the error bars refer to the $80 \%$ likelihood values discussed by KESCS (henceforth denoted by $\sigma_{80 \%}$ ) and we include arclets with $q=2$ for purposes of illustration. Although the sample of $q=1$ arclets is small, it is useful to ask at what level of significance do the results agree with the predictions. 
T.M.D. Ebbels et. al.

Figure 10. LDSS-2 spectra for arclets in Abell 2218. (Continued)

Figure 11. LDSS-2 spectra for arclets in Abell 2218. (Continued)

The arclets can be grouped according to the degree of success with which we predicted their redshifts. In total there are 11 arclets of class II and $q=1$ for which meaningful redshift predictions can be made. Of these, 9 match their spectroscopic redshifts within $2 \sigma_{80 \%}$. As tests of the method, the best of these are arclets \#231, \#297, \#317, \#381 \#467 \& \#456 which have small error bars leaving little likelihood that the predictive success could be a product of chance alone. The remaining 3 arclets (\#145, \#1581 \& \#238) have larger error bars due to their relatively large values of $\left|\tau_{y}\right|$, but still agree tolerably well with their spectroscopic redshifts. Finally there are two arclets whose predictions do not match their spectroscopic redshifts within $2 \sigma_{80 \%}$ - objects \#205 and \#464. Morphologically, \#205 is clearly a spiral galaxy (Figure 9) and this apparent lack of distortion so close to the cluster core leads to the low redshift prediction. \#464, however appears quite distorted in the direction of the local shear and its predicted redshift is correspondingly high. The disagreement between prediction and measurement for the arclets \#205 and \#464 must therefore result from the natural dispersion of image shapes on the sky, leading to chance alignments in the source plane away from and along the shear direction respectively. Given the shape of the field galaxy ellipticity distribution as illustrated by contours in Figure 12, it is not unusual to find galaxies in these regions (reasonably large $\left|\tau_{x}\right|$ but small $\left|\tau_{y}\right|$ ). Thus we conclude that these two cases represent merely a statistical fluctuation in this small sample.

We can now consider, in the light of Figure 13, the nature of the error in the predictions for the population as a whole. Taking only the $q=1$ arclets, the distribution shown in Figure 13 has a mean of $\left\langle\delta_{z} / z\right\rangle=0.07$ and dispersion $\sigma_{\delta_{z} / z}=0.48$, while including the $q=2$ arclets brings these values to $\left\langle\delta_{z} / z\right\rangle=0.09$ and $\sigma_{\delta_{z} / z}=0.41$. We therefore expect the fractional error in the predicted quantities of the whole population (such as $\langle z\rangle$ ) to be of order $10 \%$ or less, despite the dispersion of individual points.

The conclusion from the above discussion is that while $z_{\text {opt }}$ and $z_{\text {spec }}$ agree well for many of the arclets, we cannot expect this to be the case for all arclets. This is simply due to the unknown position of $\bar{\tau}$ within the natural distribution of source shapes. As mentioned above, it is useful to consider how the predicted ellipticity distribution of our sample compares with that seen in the field generally. Returning to Figure 12, the lower two panels illustrate how both the spectroscopic and fainter samples trace the field galaxy distribution (contours, measured from $\sim 10000$ galaxies between $I=18-25$ taken from HST WFPC2 fields in the MDS and Groth Strip Surveys (Ebbels et al. in prep.)). Two effects can clearly be seen. The first is the tendency in the image plane, for the spectroscopic sample (filled symbols) to be more extended than the contours. This justifies our selection of these objects as arclet candidates - they are more extended than the field population in general. The second effect is a result of the method itself, specifically that our choice of the optimum redshift of an object will correspond to its low- est obtainable ellipticity. Thus the distribution of points in the source plane will tend to be more centrally concentrated than in the field and this can readily be seen in the Figure. Notwithstanding these biases however, it is clear that our predicted distribution favors no particular orientation and is centrally peaked - the two features most noticeable about the unlensed field distribution.

In using this technique to probe the redshift distribution of faint galaxies, we are mainly concerned with certain statistical properties of this distribution such as the mean $\langle z\rangle$ and the dispersion $\sigma_{z}$. We are therefore compelled to compare the predicted values of these quantities with their actual values for our sample. We show the redshift distributions derived from the inversion method and from spectroscopy in Figure 14, where we have ensured that both distributions correspond to that subset of our sample possessing both inversion and spectroscopic redshifts. Clearly the two distributions are similar in form with the bulk of the arclets below a redshift of one. Using just those arclets with $q=1$, we find $\left\langle z_{\text {spec }}\right\rangle=0.68$ while the predicted value is $\left\langle z_{\text {opt }}\right\rangle=0.69$, a difference of just $1.5 \%$. Expanding the sample to include the $q=2$ arclets, we find $\left\langle z_{\text {spec }}\right\rangle=0.63$ and $\left\langle z_{\text {opt }}\right\rangle=0.61$, a difference of $2 \%$. In this case therefore, the mean redshift is very accurately predicted by the technique. Looking at the shape of the distributions, one may compare their dispersions. Considering the $q=1$ data, we find $\sigma_{z_{\text {spec }}}=0.49$ and $\sigma_{z_{\text {opt }}}=0.62$ while including the data with $q=2$ gives $\sigma_{z_{s p e c}}=0.42$ and $\sigma_{z_{o p t}}=0.53$. Bootstrap resampling of the true (spectroscopic) distribution indicates that the widths of the inversion and spectroscopic distributions are not statistically distinguishable at greater than $\sim 75 \%$ confidence. We do expect, however, that the uncertainties in the inversion redshifts will increase the predicted dispersion over the spectroscopic one. We may therefore consider the inversion-predicted dispersion better as an upper limit on the true width of the redshift distribution. In summary then, this analysis gives us confidence that our method can find the correct mean redshift for an unknown distribution, but that the width of the distribution is less well determined. For the mean redshift, the agreement between the predicted and spectroscopic values is especially encouraging when one considers the small sample size.

\section{IMPLICATIONS FOR THE MASS MODEL}

In this section we return to the mass model used by KESCS and investigate to what extent the new spectroscopic results further constrain it.

First, we consider only the spectroscopic redshifts of the multiple images, namely: \#359 ( $\mathrm{z}=0.702)$, \#259 ( $\mathrm{z}=1.034)$, \#384 ( $\mathrm{z}=2.515)$, and \#444 ( $\mathrm{z}=1.030)$ and optimize the different cluster and galaxy scale components following the KESCS prescription. Following the analysis of Natarajan $\&$ Kneib (1997), we also vary the exponent $\alpha$ of the scaling law of the truncation radius $\left(r_{c u t}=r_{c u t}^{*}\left(L / L_{*}\right)^{1 / \alpha}\right)$. We 
source plane, shear coords

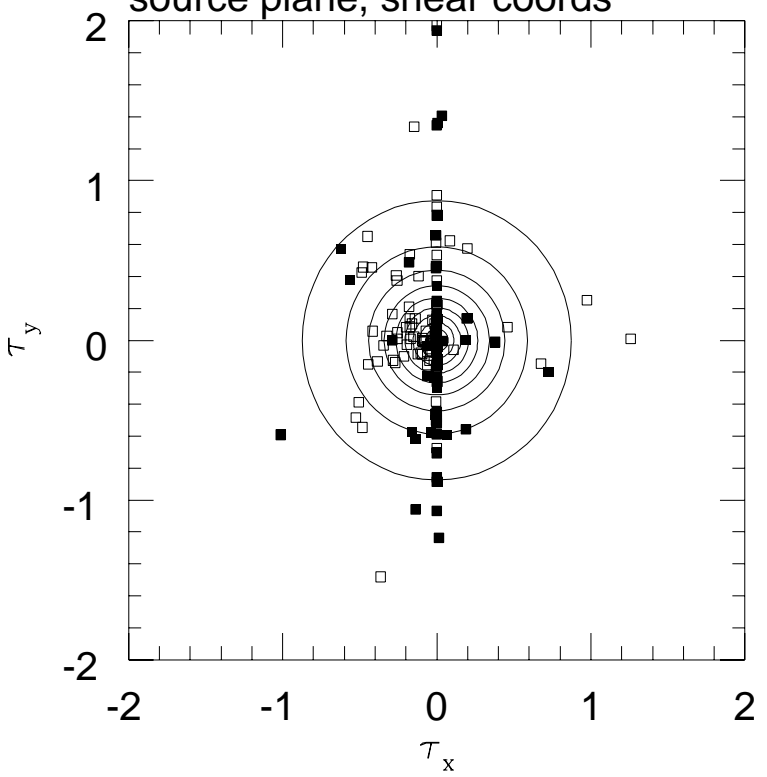

source plane, CCD coords

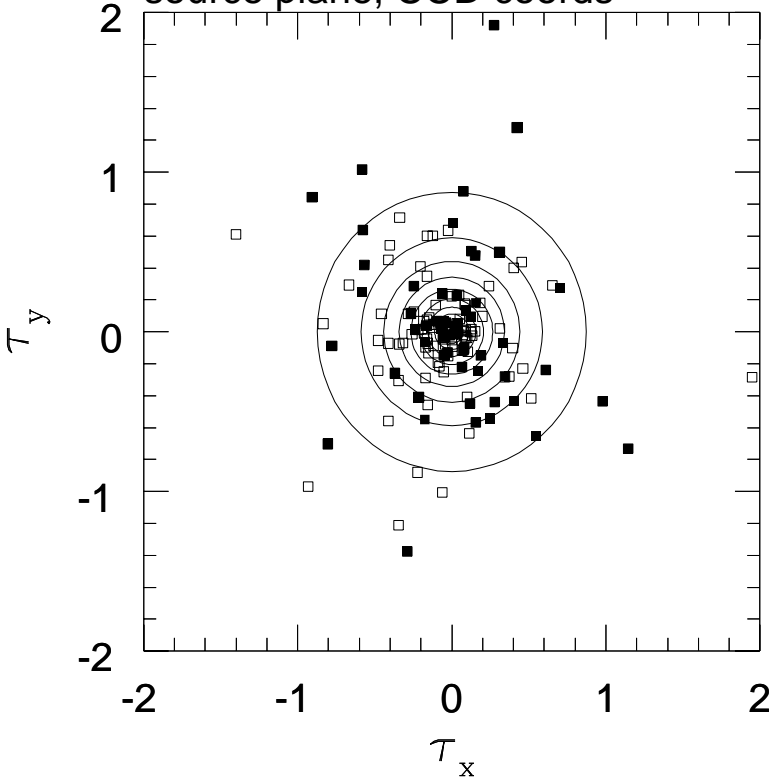

image plane, shear coords

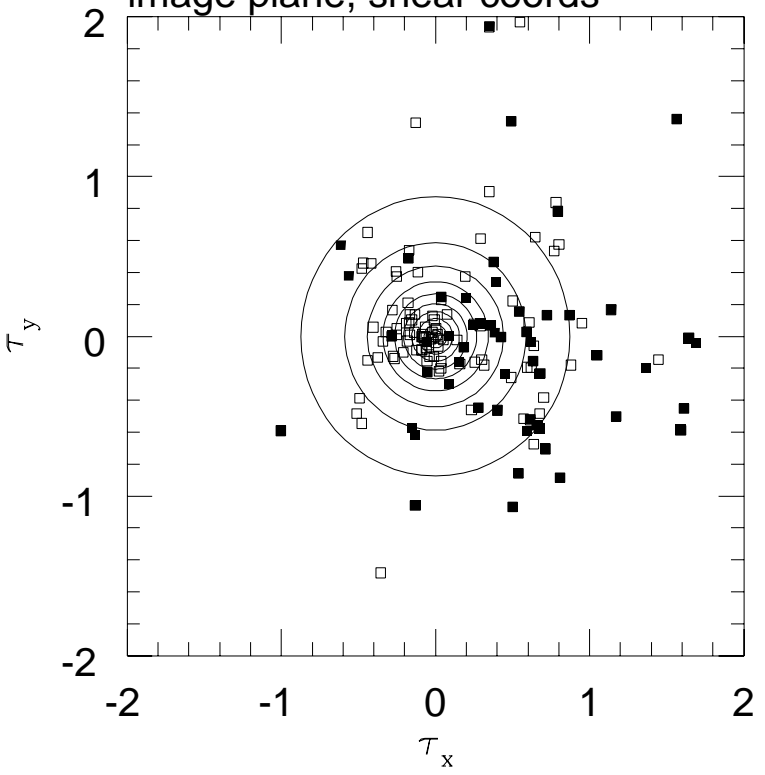

image plane, CCD coords

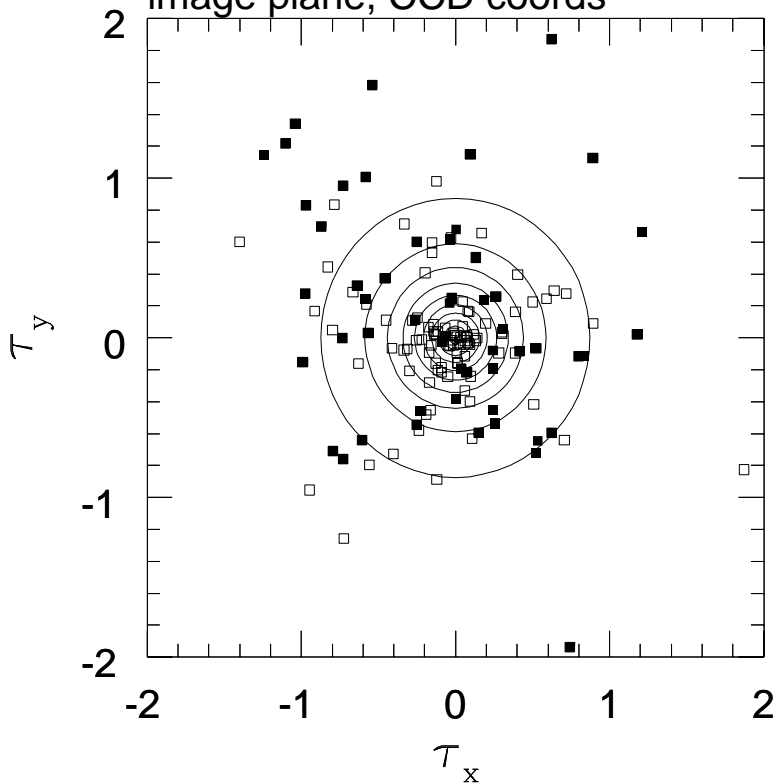

Figure 12. The lens inversion method. We show plots of the $\bar{\tau}$ ellipticity vectors for the arclets in the source plane (left panel) and the image plane (right panel). For each plane, we show the $\bar{\tau}$ vectors with respect to the frame of the local shear (upper panel) and the CCD frame (lower panel). In all plots we show linearly spaced contours of the field galaxy $\bar{\tau}$ distribution as measured from HST field survey images (Ebbels et al. in preparation). Solid symbols represent the spectroscopic sample while open ones indicate the full sample of objects with areas over 50 pixels (137 arclets.) The inversion method is clearly seen at work in the upper panel. In the image plane (top right), most of the arclets have $\tau_{x}>0$ since most of them (especially the spectroscopic sample) are aligned with the local shear. Placing each source at a redshift behind the cluster translates the corresponding point on this diagram in $\mathrm{x}$ only. For each arclet we choose that redshift at which $\tau$ is minimized, and thus the invertible arclets (class II) will end up on the line $\tau_{x}=0$ in the source plane (top left). In the source plane, class I arclets (not sufficiently distorted to invert) lie in the region $\tau_{x}<0$ and class III arclets (too elongated to invert) lie in the region $\tau_{x}>0$ as is clearly seen. Moving to the CCD frame, one can see that the distribution of points in the image plane (bottom right) is more extended than that of field galaxies in general (justifying their selection as elongated arclets.) In the source plane (bottom left) the effect is reversed since we choose the roundest shape for each object and the distribution of points is more peaked than the field. It is clear that when referred to the CCD coordinates, there is no preferential direction, even in the source plane. 

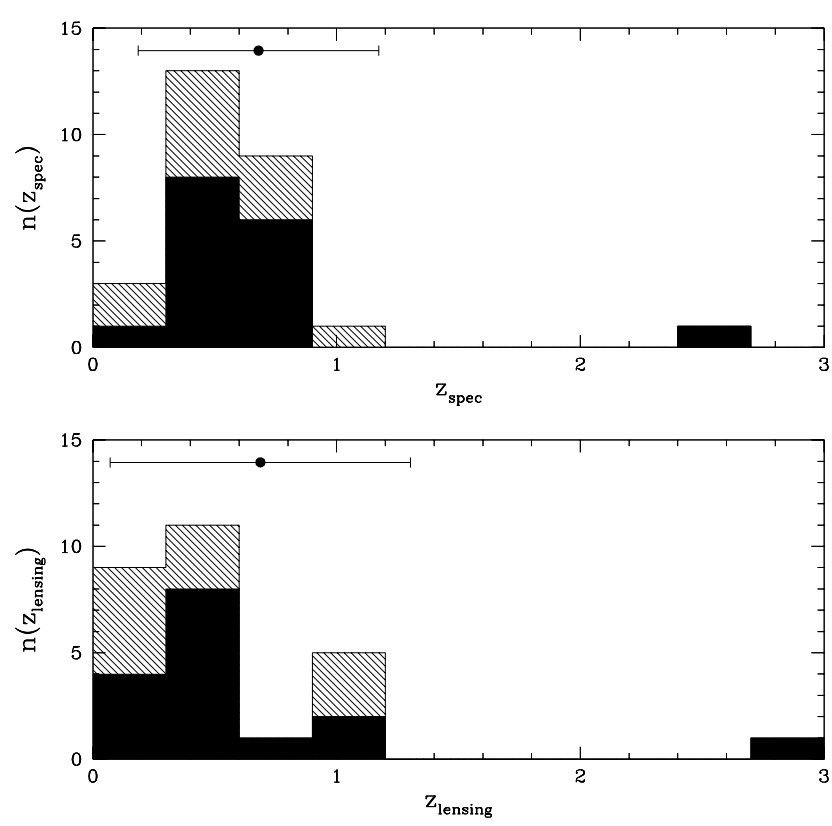

Figure 14. The distribution of our sample with spectroscopic (left) and inversion (right) redshifts. Objects with firm $(q=1)$ and tentative $(q=2)$ redshifts are denoted by solid and heavy shading shading respectively. Here we have ensured that both histograms correspond to the same sample of objects which include multiple images (except those used as model constraints). In each plot the point with error bar marks the mean and dispersion of the distribution for the $q=1$ sample.

tried two different values: $\alpha=4$ to mimic a constant mass to light ratio for the galaxies, and $\alpha=2.5$ to mimic a 'Kormendy' relation between the effective radius of the mass and the luminosity. The resulting best fit mass distribution does not differ greatly from the one presented in KESCS. Less mass is required between the two main clumps than in the KESCS model, and the $\alpha=2.5$ exponent is preferred leading to a mass-to-light ratio of 9 for an $L_{*}$ galaxy.

The improved model in turn allows us to put better constraints on the redshifts of the other multiple images. In particular, the \#H1-3 multiple arc appears to be clearly different from the arclet \#273 $(\mathrm{z}=0.80)$ as its redshift is now predicted to be $z=1.9 \pm 0.2$. In fact, closer inspection of the HST/WFPC2 image reveals a small offset between the \#273 and \#H1-3 systems of 1 pixel, suggesting that these images are indeed separate systems. The multiple system \#730 suffers the same translation to higher $\mathrm{z}$ as $\mathbf{H 1 , 2 , 3}$ and is now expected to be at a higher redshift than $\sim 3$. (With the relatively low redshift of A2218, our ability to state tighter constraints for high redshift arcs is hindered by the weak dependance on redshift of the $D_{L S} / D_{S}$ ratio.)

We then considered both the singly and multiply imaged arclets whose spectroscopic redshifts are presented here. Their images were mapped back to the source plane to compare their intrinsic shape distribution to that of the unlensed field (shown by contours in Figure 12). These intrinsic ellipticities form a circularly symmetric distribution in the $\overline{\tau_{S}}$-plane and, with few exceptions, have ellipticities within $\tau<0.2$. This area contains over half of the entire un- lensed distribution and therefore the predicted shapes of our arclets well match those expected for unlensed field galaxies. To include the single image redshifts in the optimisation, we reran the optimisation procedure, adding to the $\chi^{2}$ sum a term of the form $\left(\tau_{S} / \sigma_{\tau S}\right)^{2}$ for each arclet, where $\sigma_{\tau S}$ is the width of the intrinsic ellipticity distribution. The difference between the two optimizations is minor, demonstrating that we are now very close to the true mass distribution within the boundary of the HST/WFPC2 field.

Finally, we used the updated model on the catalogue of faint arclets with areas above 50 pixels (as used in KESCS) to compute the mean redshift versus magnitude prediction. This is shown by the thick line on Figures 15 and 16 where we compare it to the prediction of KESCS (thin lines). Clearly, while there may be some differences in $z_{\text {opt }}$ for individual arclets, the difference in the prediction for the whole sample (even in the faintest bin) is small $(\lesssim 5 \%)$. The new mass model is the best we can achieve with the present redshift constraints.

We now discuss how the residual uncertainties in the mass model can affect our redshift predictions. Of course, we can only change the mass model in such a way that it still reproduces the multiple imaging seen in the HST image. In the modelling process (see KESCS) we use a $\chi^{2}$ sum to represent how well the predicted parameters of the multiple images match those observed. Thus, in perturbing our model, we will try to keep the resulting $\chi^{2}$ as close to that of the fiducial model as possible. A number of tests may be made, corresponding to uncertainties in the model on both small and large scales.

Firstly, we may investigate the effect of the galaxy scale components. These were included in the model solely to reproduce several of the multiple image systems correctly. Only a few were actually needed to do this but a larger number $(\sim 30)$ were added corresponding to a given magnitude limit in the interests of consistency. We tested their effect on the redshift predictions by removing them at random from the model and predicting the redshift - magnitude relations, as shown in Figure 15. The triangles, circles and squares show the mean and dispersion in $\langle z\rangle$ for each bin when clumps were removed in groups of 1,2 and 5 respectively. We also show the predictions of our new model (thick line) and that published by KESCS (thin line) where both include the statistical correction for cluster and foreground contamination as described in KESCS. The only individual cases deviating significantly from the fiducial predictions were those where the galaxy scale clump corresponding to the $\mathrm{cD}$ galaxy was removed. However, since an external constraint in the form of the $\mathrm{cD}$ velocity dispersion exists for this clump, we do not consider its removal to be a valid test and exclude these cases from the analysis. In all three cases, the dispersion in $\langle z\rangle$ for each bin never rises above $\sigma_{<z>}=0.09$ and the average fractional dispersion is only $\sigma_{\langle z\rangle} /\langle z\rangle=5.6 \%$. Clearly, the mean redshift and its trend with magnitude are insensitive to the details of the mass model as represented by the galaxy scale clumps.

On large scales $(\sim 1 M p c)$, the uncertainties in the mass model derive mainly from the small angular size of the HST image. Outside the region of multiple imaging, we obtain less information on the mass profile, and features such as the extent of the main cluster clump or the presence of external mass clumps are poorly constrained. However the effect of 


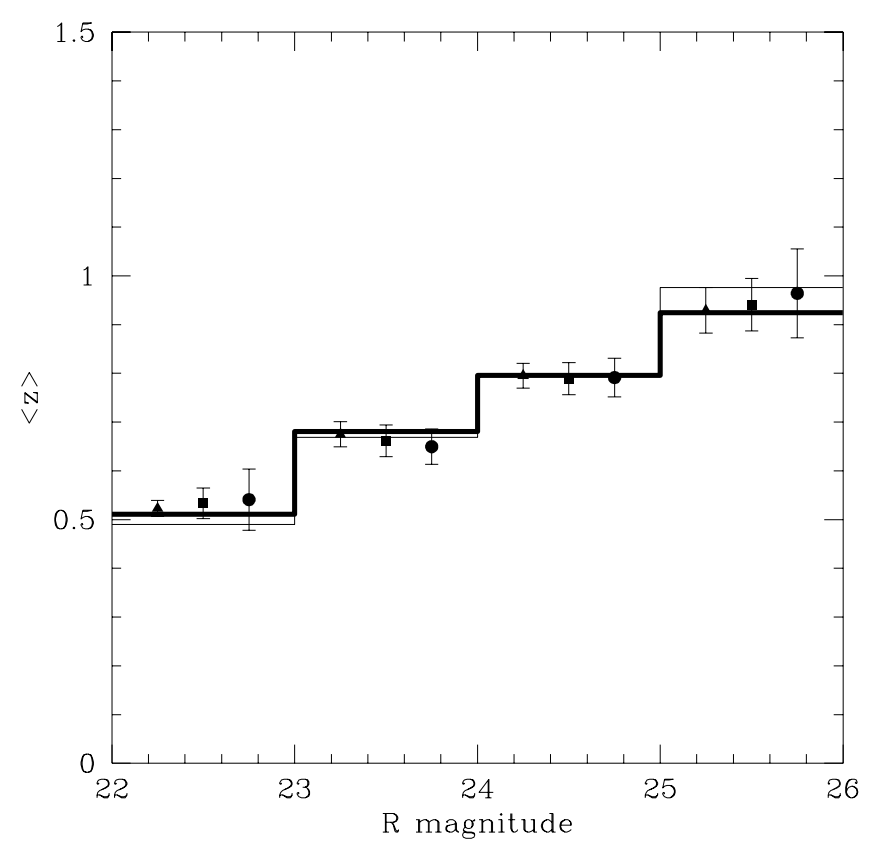

Figure 15. The effect on the redshift predictions of removing galaxy scale clumps from the model. Points with error bars show the mean and dispersion in $\langle z\rangle$ for each bin. triangles: One clump removed at a time. squares: 50 random pairs of clumps removed one at a time. circles: 50 random sets of 5 clumps removed one at a time. The three sets of points are offset in $\mathrm{R}$ for clarity. In each case, the thin line shows the mean redshift-magnitude relation for the fiducial model (KESCS) while the thick solid line shows the relation predicted by our new model developed in $\S 4$.

these uncertainties can be readily tested in a similar manner to that of the galaxy scale clumps. Figure 16 shows the effect on the magnitude-redshift relation of two such tests: adding external large scale clumps outside the HST area (squares) and varying the cut radius of the main cluster clump (circles). Again, each point shows the mean and dispersion in $\langle z\rangle$ for each bin, and we show the predictions of KESCS and our new model by thin and thick lines respectively. For the first test, large scale mass clumps varying in velocity dis-

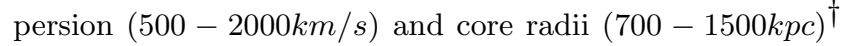
were added to the model at the same redshift as the cluster and four different positions $1.9 \mathrm{Mpc}$ from the centre of the cluster (outside the HST frame). 48 different combinations of clump parameters were tried and in each case the model was re-optimized in order to reproduce the multiple image constraints as well as possible. Only the models giving a residual $\chi^{2}$ within $30 \%$ of the fiducial model value were considered. Again, the maximum dispersion is low, $\sigma_{<z>}=0.10$ and the average fractional dispersion is just $\sigma_{<z>} /\langle z\rangle=6.4 \%$. For the second test, the cut radius of the main cluster clump, $r_{c u t}$, was varied between $400-2000 k p c$, re-optimizing the model to reproduce the multiple images as before. Here the maximum dispersion is $\sigma_{<z>}=0.04$ while the average fractional dispersion is $\sigma_{<z>} /\langle z\rangle=4.5 \%$. Once more, we find

$\dagger$ Here and in the following we assume $H_{o}=50 \mathrm{~km} \mathrm{~s}^{-1} \mathrm{Mpc}^{-1}$ and $q_{o}=0.5$

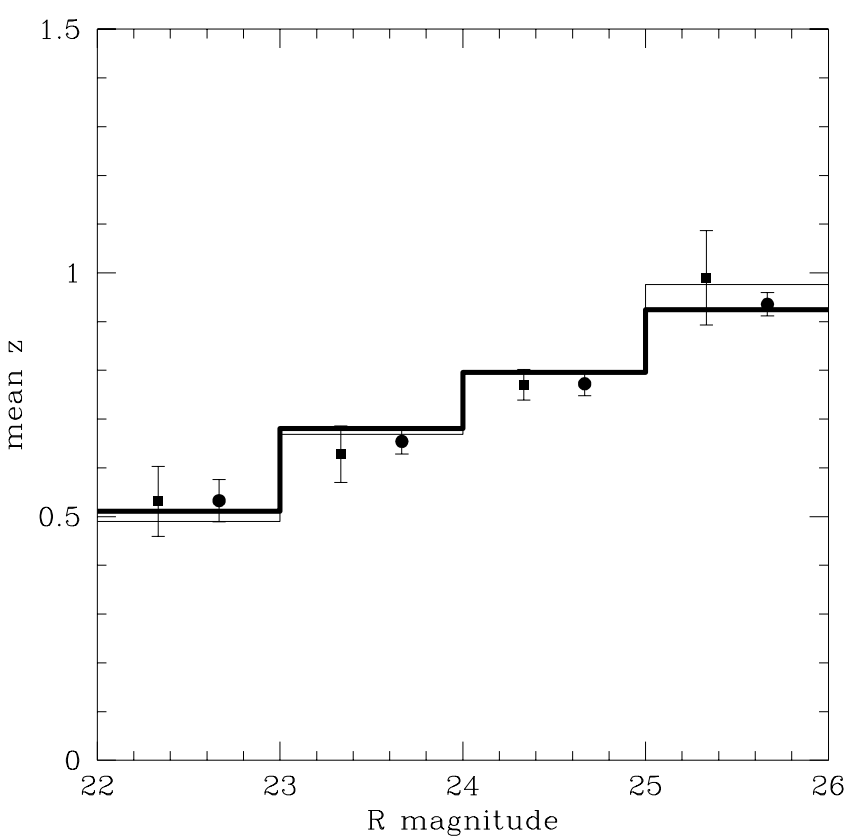

Figure 16. The effect of adding large scale external mass concentrations to the model (squares) and of varying the cut radius of the main cluster clump (circles). Each point and error bar shows the the mean and dispersion in the $\langle z\rangle$ for each bin and the points are separated in $\mathrm{R}$ for clarity as before. Again the thin solid line represents the fiducial model of KESCS while the thick line shows the predictions from our new model of $\S 4$.

that the uncertain features of our model on large scales do not affect the results appreciably. In summary then, our redshift predictions for the faint population behind A2218 are unlikely to be much affected by the remaining uncertainties in our mass model.

\section{PROPERTIES OF THE FAINT POPULATION}

The results of our spectroscopic program presented in section $\S 3$ give strong support to the inverted redshift distribution derived for fainter arclets viewed through Abell 2218. Moreover, the discussion of $\S 4$ demonstrates that further changes to the mass model permitted by our spectroscopic data will not greatly affect these redshift predictions.

For arclets with well measured shapes, such as our spectroscopic sample, we found in $\S 3$ an error in the mean redshift of $\sim 2 \%$. Using fainter arclets with more poorly measured shapes would increase the errors for each individual arclet and thus increase the uncertainty in the mean redshift for the population as a whole. This point was addressed fully in KESCS where it was shown using simulations that reliable shapes could be derived for arclets with isophotal areas above 50 pixels. We must therefore restrict ourselves to this area limit in any analysis of the population. The results (Figures 15-16) show a mean redshift $\langle z\rangle \simeq 0.8-1.0$ for amenable sources selected with $R \simeq 25.5(B \sim 26-27)$.

A low mean redshift at such a faint limit has consistently emerged from statistical lensing studies (Smail et al. 1994, KESCS). Recently, Luppino \& Kaiser (1997) have re- 
ported the detection of a significant weak shear signal from the bluest half of the faint field population behind a distant cluster, inferring a high mean redshift $(z>>1)$ for this subsample. However, we note that by combining the blue and red subsets from Luppino \& Kaiser's analysis the mean redshift they would infer for the whole faint galaxy population would be much closer to the no evolution expectation (in line with the results presented here) especially when allowance is made for the various uncertainties in their measurements and analysis.

The only independent technique which has been used to investigate the redshift distribution of galaxies beyond the spectroscopic limit is that based on multicolour photometry (the photometric redshift technique). The popularity of this technique has increased considerably of late because of precise data available for the Hubble Deep Field (HDF). It is thus of interest to compare our results with those of workers who have determined photometric redshifts in the HDF. Several surveys have been published using the HDF optical photometry combined with spectral templates derived either from population synthesis or local spectral energy distributions or both. The advantages of adding near infrared photometry to follow the usual spectral features to redshifts beyond $\sim 1.3$ are clear, but are difficult to compare with our results since they are usually magnitude limited in some infrared band. Here we compare our redshift distribution with the results of Mobasher et al. (1996) and Lanzetta et al. (1996).

Such comparisons are, however, hindered by the different techniques used and, in particular, that it is difficult to construct a magnitude limited sample from the lensing inversion method. Firstly, since the magnification of images varies across the cluster, our observed magnitude limit does not correspond to a sharp intrinsic magnitude limit, rather the lensing sample suffers from a more gradual fall off in counts with magnitude. One approach would therefore be to choose an effective magnitude limit corresponding to the point where the counts fall significantly below those in the field. The second complication arises from the area limit discussed above. In this case, we can choose to select the same proportion of faint galaxies as a function of area from both the lensing and photometric redshift samples. These choices correspond to first making a magnitude cut at $R \simeq 24$ $(I \simeq 23.5)$ and then taking only the largest $59 \%$ of the sample at this limit. Figure 17 shows the comparisons, where we have chosen to use a slightly deeper magnitude limit $(I<24)$ for the photometric redshift distributions in order to obtain a comparable number of galaxies from the HDF.

It can readily be seen that the agreement is much better when we compare to the results of Mobasher et al. than Lanzetta et al. (although the numbers of galaxies are small.) Excluding the galaxies below $z=0.2$ (which cannot be probed by our lensing analysis), we find the lensing $n(z)$ has a median of $z=0.4$ with only $7 \%$ of objects appearing above $z=1.5$ while Mobasher et al.'s distribution has a slightly higher median redshift $(z=0.5)$ but with a longer tail (19\% above $z=1.5$ ). The corresponding numbers for the Lanzetta et al. distribution are $z=0.8$ and $18 \%$. However, as one goes fainter, the agreement worsens. Figure 18 shows the predicted redshift distributions for our faintest magnitude slice $24<R<25.5$. At this limit, the lensing method predicts a low median redshift of $z=0.7$ while the
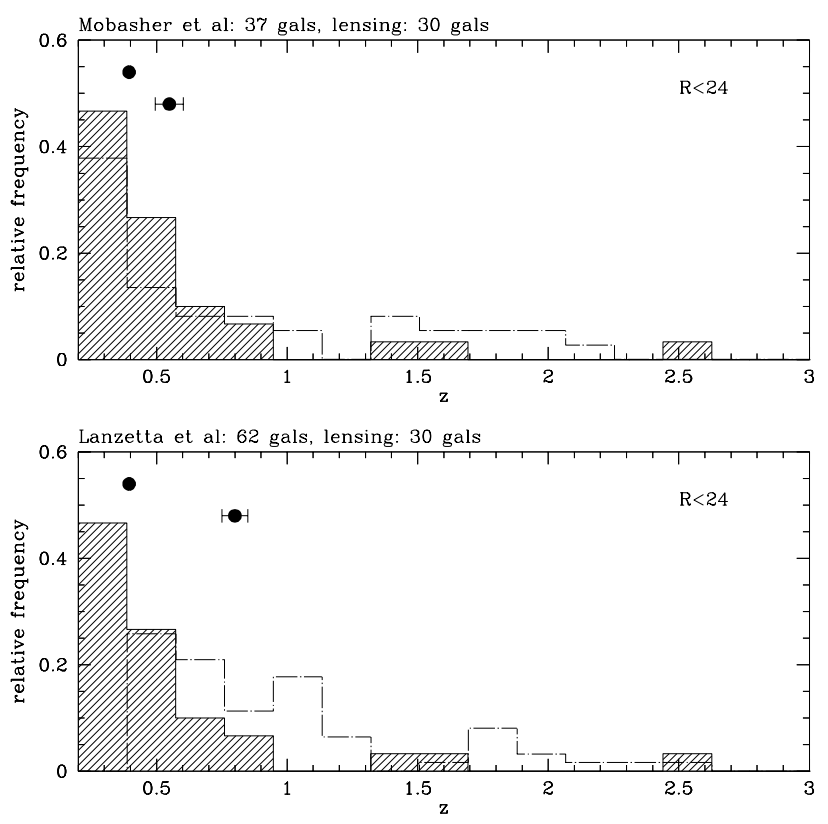

Figure 17. Comparison of the lensing $n(z)$ (shaded) with the photometric $n(z)$ from the Hubble Deep Field analyses of Mobasher et al (top) and Lanzetta et al. (bottom) for the magnitude limit $R<24$. Points with errorbars show the median of each distribution, with errors calculated from bootstrap resampling. In order to make a valid comparison, only the largest $59 \%$ of objects were used. It is clear that there is much closer agreement with Mobasher et al than Lanzetta et al, emphasizing the discrepancies between the two photometric $\mathrm{n}(\mathrm{z})$ determinations.

corresponding values from optical multi-colour photometry are $z=0.9$ (Mobasher et al.) and $z=1.1$ (Lanzetta et al.) respectively.

At the faintest limits, it is important to bear in mind that the sensitivity of photometric redshifts in the $1<z<2$ region is expected to be very poor as there are no significant continuum features at optical wavelengths. For $z>1.3$, the $4000 \AA$ break disappears from the optical window and the Lyman break does not enter until $z>2$. Is it therefore possible that some galaxies allocated to $1.5<z<2.0$ by the photometric technique may actually lie at lower redshifts ?

An important recent development in this regard is the addition of JHK ground-based near-infrared data to complement the earlier optical photometric redshift studies. Connolly et al. (in prep.) report a redshift distribution based on 4-colour optical plus 3-colour near infrared data for a $J<23.5$ sample which has $\langle z\rangle=0.9 \pm 0.1$. This corresponds to a limit somewhat deeper than $R=25.5$ but is a good indication of the changes that we might expect when nearinfrared data is added to the studies discussed above.

\section{CONCLUSIONS}

We summarize our conclusions as follows:

- We have conducted one of the deepest spectroscopic surveys attempted on a $4 \mathrm{~m}$ class telescope and certainly the faintest with LDSS-2. The faintest redshifts were obtained 

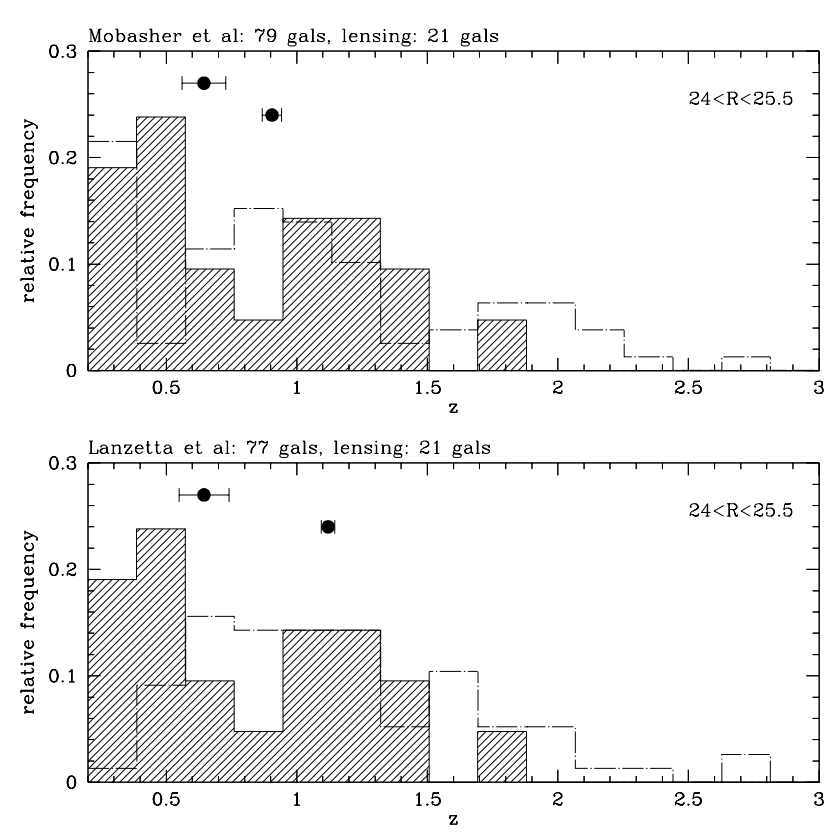

Figure 18. Comparison of the lensing $n(z)$ (shaded) with the photometric $n(z)$ of Mobasher et al. (top) and Lanzetta et al (bottom) for the magnitude slice $24<R<25.5(23.5<I<25)$. Again, points with errorbars show the median of each distribution, and only the largest $59 \%$ of objects used. The lens inversion method predicts a significantly lower median redshift than those based on optical multi-colour photometry at these limits.

for sources with $R=24$ whose unlensed magnitudes reach $R=25$ in some cases. The major limiting factor is the effectiveness of sky subtraction and our limit is more appropriately constrained by surface brightness than integrated magnitude. We have achieved a useful success rate to $\mu_{R}=24$ $\operatorname{arcsec}^{-2}$ with completeness falling below $50 \%$ at $\mu_{R}=23$ $\operatorname{arcsec}^{-2}$.

- The results of our spectroscopic survey show that the lensing inversion technique is reliable. The mean redshift of our spectroscopic sample determined from both inversion and spectroscopic redshifts agree to within $\sim 2 \%$. Since the inversion method is purely geometric in operation, this agreement gives us confidence when estimating the mean redshift of the larger population of faint arclets provided their individual shapes can be adequately measured.

- We have included the new spectroscopic redshifts to further constrain our mass model and find that the redshift predictions change by a maximum of $\sim 5 \%$. Moreover, the predicted redshifts for a population of arclets with $R \simeq 25.5$ are insensitive to further changes in the mass model allowed by the multiple image constraints. We conclude that errors in the inferred mean redshift deriving from uncertainties in the mass distribution can remain only below the level of $\delta_{<z>} /\langle z>\lesssim 10 \%$.

- The new predictions for the faint population do not differ significantly from those presented by KESCS. The mean redshift for the population of arclets amenable to inversion with $R \simeq 25.5$ is broadly consistent with the no evolution prediction; we find $\langle z\rangle \simeq 0.8-1.0$.

- To a limit set by our magnitude and area cuts, the in- version prediction agrees reasonably well with results presented by other authors using photometric redshifts determined from data on the Hubble Deep Field. A direct comparison is difficult since the inversion technique requires an area limited survey in contrast to one that is magnitude limited. The agreement may be much better when near-infrared photometry is used in addition to optical colours. This can be understood via a greater sensitivity to the $4000 \AA$ break which is redshifted into the near-infrared at redshifts of interest.

Application of this technique to other clusters with similar data has already begun (Kneib et al. in prep.). This will generate a much larger sample of inverted arclets and hence beat down the statistical uncertainties, as well as overcoming the effects of redshift clustering in the background population. Using cluster lenses over a wide range of redshifts will reduce the intrinsic uncertainties coming from the redshift dependence of the distance ratio, while extending the technique into the near-infrared using NICMOS will allow us to select $1<z<3$ galaxies in their restframe optical, to provide a more robust measure of the star-formation density at these epochs. By expanding our data set in this way, we shall be able to use this proven technique to derive robust and useful parameters for the faint population of galaxies at depths presently unobtainable by conventional means.

\section{ACKNOWLEDGMENTS}

We thank Mike Breare and all the La Palma support staff for ensuring the smooth operation of LDSS-2 on the WHT. We especially thank Karl Glazebrook for the use of his $R E D$ SHIFT utility. We thank Nial Tanvir and Karl Glazebrook for their expertise with both LDSS-2 and the LEXT software and we thank Bernhard Geiger for useful discussions on the inversion method. TMDE and IRS acknowledge financial support from PPARC.

\section{REFERENCES}

Allington-Smith J., Breare M., Ellis R.S., Gellatly D., Glazebrook K., Jorden P., Maclean J., Oates P., Shaw G. \& Tanvir N., 1994, PASP, 106, 983.

Colless M., Ellis R.S., Taylor K., \& Hook R.N., 1990, MNRAS, 253,686 .

Connolly, A.J., Szalay, A.S., Dickinson, M.E., SubbaRao, M. \& Brunner, R., in preparation.

Cowie L.L., Hu E.M., \& Songaila A., 1995, Nature 377, 603.

Cowie L.L., Songaila, A., Hu., E.M. \& Cohen, J.G., 1996, AJ, $112,839$.

Ebbels T.M.D., Le Borgne, J.-F., Pelló, R., Ellis R.S., Kneib J.-P., Smail, I., \& Sanahuja, B., 1996, MNRAS, 281, L75.

Ebbels T.M.D., Ellis R.S., Kneib J.-P., Pelló, R., \& Le Borgne, J.-F., in preparation.

Glazebrook, K., Ellis, R.S., Colless, M., Broadhurst, T., Allington-Smith, J. \& Tanvir, N., 1995, MNRAS, 273, 157.

Holtzman, J.A., Burrows, C.J, Casertano, S., Hester, J.J, Trauger, J.T., Watson, A.M. \& Worthey, G., 1995, PASP, $107,1065$.

Kneib, J.-P., Mellier, Y., Fort, B. \& Mathez, G., 1993, A\&A, 273, 367.

Kneib, J.-P., Mathez, G., Fort, B., Mellier, Y., Soucail, G. \& Longaretti, P.-Y., 1994, A\&A, 286, 701. 
Kneib, J.-P., Ellis, R.S., Smail, I., Couch, W.J., Sharples, R., ApJ, 471, 643. (KESCS)

Kneib, J.-P., Pelló, R., Ellis, R.S., Smail, I., Le Borgne, J.-F., in preparation.

Lanzetta, K.M., Yahil, A. \& Fernández-Soto, A., 1996, preprint astro-ph 9606171

Le Borgne, J.-F., Pelló, R. \& Sanahuja, B., 1992, A\&AS, 95, 87.

Luppino, G.A. \& Kaiser, N., 1997, ApJ, 475, 20.

Mellier, Y., Fort, B., \& Kneib, J.-P., 1993, ApJ, 407, 33

Mobasher, B., Rowan-Robinson, M., Georgakakis, A. \& Eaton, N., 1996, MNRAS, 282, 7.

Pelló R., Le Borgne, J.-F., Sanahuja, B., Mathez G., \& Fort B., 1992, A\&A, 266, 6 .

Smail, I., Ellis, R.S., \& Fitchett, M.J., 1994, MNRAS, 270, 24.

Smail, I., Ellis, R.S., Fitchett, M.J. \& Edge, A.C., 1995, MNRAS, 273, 277.

Smail, I., Couch, W.J., Ellis, R.S. \& Sharples, R., 1995, ApJ, 440, 501.

Steidel, C.C., Giavalisco, M., Pettini, M., Dickinson, M. \& Adelberger, K.L., 1996, ApJL, 462, L17. 